\title{
Analysis of Kolmogorov Flow and Rayleigh-Bénard Convection using Persistent Homology
}

\author{
Miroslav Kramár ${ }^{\mathrm{a}}$, Rachel Levanger ${ }^{\mathrm{a}}$, Jeffrey Tithof ${ }^{\mathrm{b}}$, Balachandra Suri ${ }^{\mathrm{b}}, \mathrm{Mu}$ Xuc ${ }^{\mathrm{c}}$, Mark Paul ${ }^{\mathrm{c}}$, Michael F. Schatz ${ }^{\mathrm{b}}$, \\ Konstantin Mischaikow ${ }^{\mathrm{a}}$ \\ ${ }^{a}$ Department of Mathematics, Hill Center-Busch Campus, Rutgers University, 110 Frelingheusen Rd, Piscataway, NJ 08854-8019, USA \\ ${ }^{b}$ Center for Nonlinear Science and School of Physics, Georgia Institute of Technology, Atlanta, GA 30332-0430, USA \\ ${ }^{c}$ Department of Mechanical Engineering, Virginia Tech, Blacksburg, VA 24061, USA
}

\begin{abstract}
We use persistent homology to build a quantitative understanding of large complex systems that are driven far-fromequilibrium. In particular, we analyze image time series of flow field patterns from numerical simulations of two important problems in fluid dynamics: Kolmogorov flow and Rayleigh-Bénard convection. For each image we compute a persistence diagram to yield a reduced description of the flow field; by applying different metrics to the space of persistence diagrams, we relate characteristic features in persistence diagrams to the geometry of the corresponding flow patterns. We also examine the dynamics of the flow patterns by a second application of persistent homology to the time series of persistence diagrams. We demonstrate that persistent homology provides an effective method both for quotienting out symmetries in families of solutions and for identifying multiscale recurrent dynamics. Our approach is quite general and it is anticipated to be applicable to a broad range of open problems exhibiting complex spatio-temporal behavior.
\end{abstract}

\section{Introduction}

We introduce new mathematical techniques for analyzing complex spatiotemporal nonlinear dynamics and demonstrate their efficacy in problems from two different paradigms in hydrodynamics. Our approach employs methods from algebraic topology; earlier efforts have shown that computing the homology of topological spaces associated to scalar or vector fields generated by complex systems can provide new insights into dynamics $[1,[2,3,[4,[5,[6]$. We extend prior work by using a relatively new tool called persistent homology [7, 8, 9].

Complex spatiotemporal systems often exhibit complicated pattern evolution. The patterns are given by scalar or vector fields representing the state of the system under study. Persistent homology can be viewed as a map PD that assigns to every field a collection of points in $\mathbb{R}^{2}$, called a persistence diagram. For a given scalar field $f: D \rightarrow R$, the points in the persistence diagram $\mathrm{PD}(f)=\left\{\mathrm{PD}_{k}(f)\right\}_{k=0}^{\infty}$ encode geometric features of the sub-level sets $\mathrm{C}(f, \theta)=\{x \in D \mid f(x) \leq \theta\}$ for all values of $\theta$. A feature encoded by the point $\left(\theta_{b}, \theta_{d}\right) \in \mathrm{PD}_{k}(f)$ represents a feature at the $k$-th homology level that appears in $\mathrm{C}\left(f, \theta_{b}\right)$ for the first time and disappears in $\mathrm{C}\left(f, \theta_{d}\right)$.

Email addresses: miroslav@math.rutgers.edu (Miroslav Kramár), rachel@math.rutgers.edu (Rachel Levanger), jtithof3@gatech.edu (Jeffrey Tithof), balachandra@gatech.edu (Balachandra Suri), xumu8621@vt.edu (Mu Xu), mrp@vt.edu (Mark Paul), michael.schatz@physics.gatech.edu (Michael F. Schatz), mischaik@math.rutgers.edu (Konstantin Mischaikow)

Preprint submitted to Elsevier
Therefore, $\theta_{b}$ and $\theta_{d}$ are called birth and death coordinates of this feature. The lifespan $\theta_{d}-\theta_{b}>0$ indicates the prominence of the feature. In particular, features with long lifespans are considered important and features with short lifespans are often associated with noise. Thus, the persistence diagram is a highly simplified representation of the field generating the pattern.

The space of all persistence diagrams, Per, can be endowed with a variety of metrics under which PD is a Lipschitz function. This has several important implications that we exploit in this paper. First, the Lipschitz property implies that small changes in the field pattern, e.g. bounded errors associated with measurements or numerical approximations, lead to small changes in the persistence diagrams. Second, by using different metrics, we can vary our focus of interest between larger and smaller changes in the persistence diagrams. Moreover, by comparing different metrics, we can infer if the changes in a pattern affect geometric features with longer or shorter life spans. Finally, since, applying the map PD to a time series of patterns produces a time series in Per, the distance between the consecutive data points in Per can be used to quantify the average rate at which the geometry of the patterns is changing.

As mentioned above, the dynamics of spatiotemporal systems are characterized by the time-evolution of the patterns corresponding to the fields generated by the system. However, capturing these vector fields, either experimentally or numerically, results in multi-scale high dimensional data sets. In order to efficiently analyze these data sets, 
a dimension reduction must be performed. We use persistent homology to perform nonlinear dimension reduction from a time series of patterns to a time series of persistence diagrams. We show that this reduction can cope with redundancies introduced by symmetries (both discrete and continuous) present in the system. In particular, this approach directly quotients out symmetries and, thereby, permits easy identification of solutions that lie on a group orbit. Alternative approaches to nonlinear dimension and symmetry reduction include both the method of slices [10] and recent advances in identifying unstable exact solutions of nonlinear partial differential equations [11] . While a detailed comparison of these methods is beyond the scope of this paper, it is worth pointing out that the application of persistent homology does not rely on knowledge of the underlying governing equations.

Separately, we also apply persistent homology to extract information about dynamical structures in the reduced data. Characterizing dynamics in the space of persistence diagrams cannot be done using conventional methods (e.g., time delay embeddings), since choosing a coordinate system in Per is currently an open problem [12]. However, since Per is a metric space, the geometry of the point cloud $X$, generated by the time series of the reduced data, is encoded by a scalar field which assigns to each point in Per its distance to $X$. We show how persistent homology may be applied to describe dynamics by characterizing the geometry of $X$.

An outline of the paper is as follows. In Section 2 we present a brief overview of the two fluid flows examined in this paper: (1) Kolmogorov flow and (2) Rayleigh-Bénard convection. We note here, for emphasis, that while persistent homology can be applied to vector fields, it will be sufficient for this paper to focus on scalar fields drawn from these systems (specifically, one component of the vorticity field for Kolmogorov flow, and the temperature field for Rayleigh-Bénard convection).

In Section 3 we discuss key issues related to the application of persistent homology. By now, the mathematical theory of persistent homology is well developed. Therefore, our main emphasis is on the computational aspect of passing from the data to the persistence diagrams. Section 4 describes the correspondence between the geometric features of a scalar field and the points in its corresponding persistence diagram. Section [5] discusses the structure of the space Per and the properties of the associated metrics.

In Sections 6 and 7 we discuss how these metrics can be used to analyze dynamics. First, we interpret distance between the persistence diagrams representing the consecutive data points in the time series as a rate at which geometry of the corresponding scalar fields is changing. Second, we motivate and explain the procedure for extracting the geometric structure of the point cloud in Per.

We close the paper by applying the developed techniques to the following problems. In Section 8 , we identify distinct classes of symmetry-related equilibria for Kolomogorov flow. In Section 9] we show that a relative peri- odic orbit for Kolmogorov flow collapses to a closed loop in Per. Finally, in Section 10, we deal with identifying recurrent dynamics that occur on different time scales in our study of Rayleigh-Bénard convection flow.

\section{The Systems to be Studied}

\subsection{Kolmogorov Flow}

For the study of turbulence in two dimensions, Kolmogorov proposed a model flow where the evolution of a two-dimensional (2D) velocity field $\mathbf{u}(x, y, t)$ is given by

$$
\begin{aligned}
\frac{\partial \mathbf{u}}{\partial t}+\beta \mathbf{u} \cdot \nabla \mathbf{u} & =-\frac{1}{\rho} \nabla p+\nu \nabla^{2} \mathbf{u}-\alpha \mathbf{u}+\mathbf{f} \\
\nabla \cdot \mathbf{u} & =0
\end{aligned}
$$

(with $\beta=1$ and $\alpha=0$ ). In the above equation, $p(x, y)$ is the pressure field, $\nu$ is the kinematic viscosity, $\rho$ is fluid density, and $\mathbf{f}=\chi \sin (\kappa y) \hat{\mathbf{x}}$ is the forcing that drives the flow [13]. Laboratory experiments in electromagneticallydriven shallow layers of electrolyte can exhibit flow dynamics that are well-described by Equations (1) with appropriate choices of $\beta$ and $\alpha$ to capture three-dimensional effects, which are commonly present in experiments [14]. In this paper, we refer to all models described by Equations (1) (including experimentally-realistic versions) as Kolmogorov flows.

It is convenient to use the vorticity-stream function formulation [15] to study Kolmogorov flow analytically and numerically. Equations (11), written in terms of the zcomponent of the vorticity field $\omega=(\nabla \times \mathbf{u}) \cdot \hat{\mathbf{k}}$, a scalar field, take the form

$$
\frac{\partial \omega}{\partial t}+\beta \mathbf{u} \cdot \nabla \omega=\nu \nabla^{2} \omega-\alpha \omega+\chi \kappa \cos (\kappa y) .
$$

For the current study, we choose $\beta=0.83, \nu=3.26 \times$ $10^{-6} \mathrm{~m}^{2} / \mathrm{s}, \alpha=0.063 \mathrm{~s}^{-1}, \rho=959 \mathrm{~kg} / \mathrm{m}^{3}$, and $\lambda=$ $2 \pi / \kappa=0.0254 \mathrm{~m}$. We express the strength of the forcing in terms of a non-dimensional parameter, the Reynolds number $R e=\sqrt{\frac{\lambda^{3} \chi}{8 \nu^{2}}}$.

Equation (2) is solved numerically by using a semidiscrete, pseudo-spectral method [16], assuming periodic boundary conditions in both $x$ and $y$ directions, i.e., $\omega(x, y)=\omega\left(x+L_{x}, y\right)=\omega\left(x, y+L_{y}\right)$, where $L_{x}=0.085 \mathrm{~m}$ and $L_{y}=4 \lambda=0.1016 \mathrm{~m}$ are the dimensions of the domain in the $x$ and $y$ directions, respectively. The vorticity field is discretized in the Fourier space using $128 \times 128$ modes, which corresponds to spatially resolving the domain on a 2D mesh with spacing $\Delta x=L_{x} / 128$ and $\Delta y=L_{y} / 128$ in the $x$ and $y$ directions, respectively. A time step of $d t=1 / 32$ is chosen for the temporal discretization.

It is important to note that Equation (2), with periodic boundary conditions, is invariant under any combination of three distinct coordinate transformations: (1) a translation along $x: \mathcal{T}_{\delta x}(x, y)=(x+\delta x, y), \delta x \in\left[0, L_{x}\right] ;(2)$ 


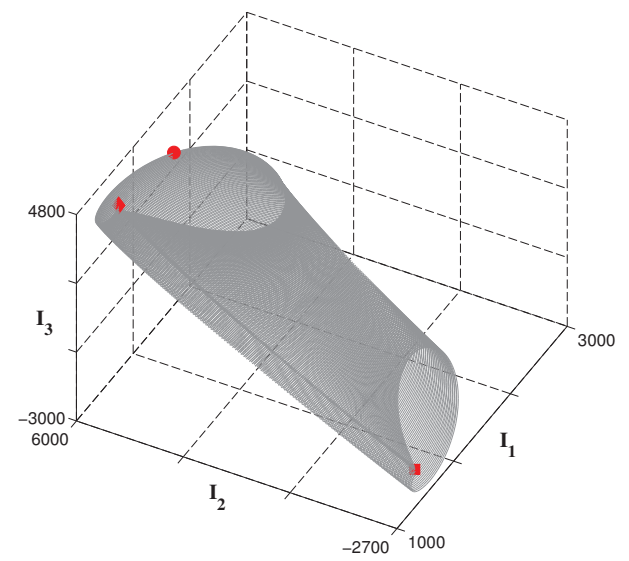

(a)

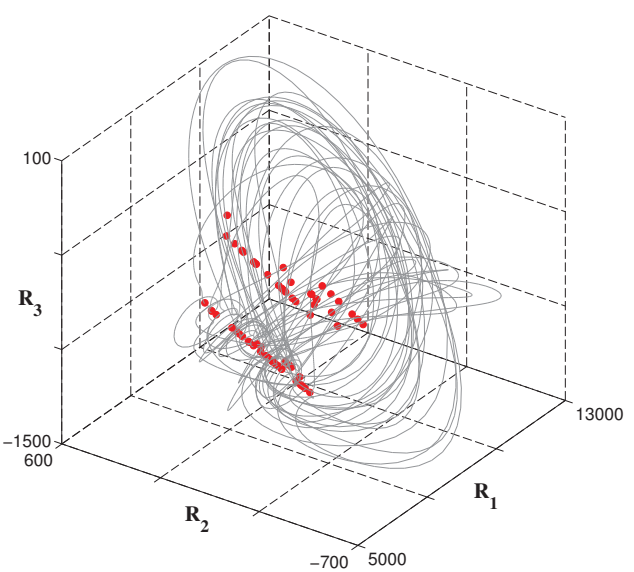

(b)

Figure 1: (a) Three-dimensional projections of a stable RPO at $R e=$ 25.43 from the Kolmogorov flow using the imaginary part of the three dominant Fourier modes, $I_{1}, I_{2}$, and $I_{3}$. The gray line indicates the evolution of a RPO; three snapshots sampled from that orbit are indicated by a red diamond, a red circle, and a red square, which are analyzed below. (b) Three-dimensional projections of a turbulent trajectory, at $R e=26.43$, using the real parts of the three dominant Fourier modes, $R_{1}, R_{2}$, and $R_{3}$. The gray line indicates the chaotic evolution of the flow, which is influenced by the presence of unstable fixed points, indicated by red circles, which are also analyzed below.

a rotation by $\pi: \mathcal{R}(x, y)=(-x,-y)$; and (3) a reflection and a shift: $\mathcal{D}(x, y)=(-x, y+\lambda / 2)$. Because of these symmetries, each particular solution to Equation (2) generates a set of solutions which are dynamically equivalent. Physically, invariance under continuous translation leads to the existence of relative equilibria (REQ) and relative periodic orbit (RPO) solutions, in addition to equilibria (EQ) and periodic orbit (PO) solutions.

For $R e=25.43$, the flow is characterized by a steady RPO; Figure 1(a) shows a projection, plotted using the three dominant Fourier modes of this RPO. The RPO has a period 34.78 seconds and a drift speed $1.354 \times 10^{-6} \mathrm{~m} / \mathrm{s}$. The tunnel-like structure is a result of the periodic motion superposed over the slow drift along the $x$-direction. For larger forcing $(R e=26.43)$, the flow becomes weakly

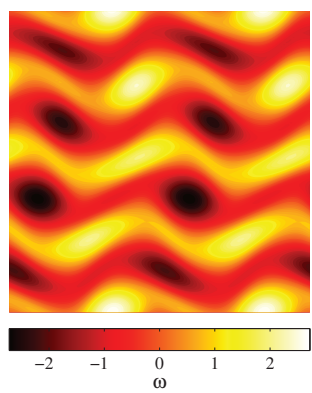

(a)

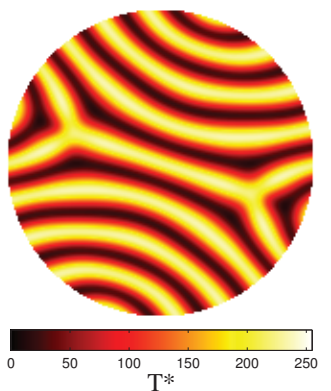

(b)
Figure 2: (a) A snapshot of the $z$-component of the vorticity field $\omega$ for Kolmogorov flow from the stable relative periodic orbit found at $R e=25.43$. (b) A snapshot of the renormalized 8-bit mid-plane temperature field $T^{*}$ for Rayleigh-Bénard convection from the stable almost-periodic orbit found at $R a=3000$ and $\operatorname{Pr}=1$.

turbulent, as can be seen from the Fourier projections in Figure 1(b). The turbulent dynamics in this regime are of great interest as the flow explores a region of the state space which contains "weakly" unstable EQ, PO, REQ, and RPO solutions. Recent theoretical advances have shown that the identification of these solutions could aid the understanding of weakly turbulent dynamics [17]. For instance, if the turbulent trajectory is close to an EQ solution $\left(\omega_{0}\right)$, which is characterized by $\partial \omega_{0} / \partial t=0$, we would expect the instantaneous rate of change of $\omega$ to be relatively small, i.e., $\partial \omega / \partial t \approx 0$. Similarly, a close pass to a PO solution would mean $\omega(t+T) \approx \omega(t)$, where $T$ is the period of the PO that is guiding the dynamics of the turbulent trajectory. The turbulent trajectory depicted in Figure 1(b) passes close to unstable EQ and REQ solutions which are indicated by the red dots.

\subsection{Rayleigh-Bénard Convection}

Rayleigh-Bénard convection is a canonical pattern forming system that has been used to gain many new fundamental insights into the spatiotemporal dynamics of systems that are driven far-from-equilibrium 18, 19]. Rayleigh-Bénard convection is the buoyancy driven fluid flow that occurs when a shallow layer of fluid is heated uniformly from below in a gravitational field. The dynamics are governed by the Boussinesq equations,

$$
\begin{aligned}
\operatorname{Pr}^{-1}\left(\frac{\partial \mathbf{u}}{\partial t}+\mathbf{u} \cdot \nabla \mathbf{u}\right) & =-\nabla p+\nabla^{2} \mathbf{u}+\operatorname{RaT} \hat{\mathbf{z}} \\
\frac{\partial T}{\partial t}+\mathbf{u} \cdot \nabla T & =\nabla^{2} T \\
\nabla \cdot \mathbf{u} & =0
\end{aligned}
$$

where $\mathbf{u}(x, y, z, t)$ is a vector field of the fluid velocity, $p(x, y, z, t)$ is the pressure field, and $T(x, y, z, t)$ is the temperature field. In our notation, the origin of the Cartesian coordinates $(x, y, z)$ at the center of the domain are at the 
lower heated plate where $\hat{\mathbf{z}}$ is a unit vector opposing gravity. Equations (3)-(5) represent the conservation of momentum, energy, and mass, respectively. The equations have been nondimensionalized using the vertical diffusion time of heat as the time scale, the layer depth as the length scale, and the constant temperature difference between the lower and upper plates as the temperature scale.

In our work, we consider Rayleigh-Bénard convection in a shallow domain with a cylindrical cross-section. The no-slip fluid boundary condition $\mathbf{u}=0$ is applied to all material surfaces. The lower and upper plates are held at a constant temperature where $T(z=0)=1$ and $T(z=$ $1)=0$, respectively. The lateral sidewalls of the cylindrical container are assumed to be perfectly conducting, which yields $T(z)=1-z$.

The dynamics can be described using three nondimensional parameters. The Rayleigh number $R a$ represents the ratio of buoyancy to viscous forces. At the critical value $R a_{c}=1708$, an infinite layer of fluid undergoes a bifurcation to straight and parallel convection rolls. For increasing values of the Rayleigh number $R a>R a_{c}$, the dynamics become periodic, chaotic, and eventually turbulent. The Prandtl number $P r$ is the ratio of the momentum and thermal diffusivities. For typical experiments using compressed gasses, $\operatorname{Pr} \approx 1$. Lastly, the aspect ratio of the cylindrical domain $\Gamma$ is the ratio of the domain's radius to its depth.

We numerically integrate Eqs. (3)-(5) using a highly parallel spectral element algorithm that has been tailored for the study of convection (c.f. [20]). Figure 2(b) shows a typical pattern from a numerical simulation of RayleighBénard convection. In this simulation, $R a=3000, \operatorname{Pr}=$ 1 , and the aspect ratio of the domain is $\Gamma=10$. The numerical simulation is initiated from a field of small random perturbations to the temperature field and is integrated for long times. Figure 2(b) illustrates the fluid temperature field at the horizontal mid-plane $(z=1 / 2)$, where light is warm rising fluid and dark is cool falling fluid. This image is a snap shot in time of a time-dependent pattern where the dynamics are nearly periodic in time. The pattern shown does not include the region near the sidewall. Specifically, a distance of one-layer depth from the lateral sidewall is not shown (this distance is approximately the width of a convection roll). This is done to remove the complex fluid flow that occurs in the small region near the sidewalls to allow our diagnostics to focus upon the bulk patterns and dynamics (c.f. [19]).

\section{Persistent Homology}

The aim of this paper is to introduce an approach for analyzing the dynamics of the pattern evolution in spatiotemporal systems. This is done in two steps. First, we perform nonlinear data reduction, and then we extract information about the dynamical structures from this reduced data. We formulate both of these tasks in terms of analyzing the structure of the sub-level sets of a scalar function $f: D \rightarrow \mathbb{R}$, where $D$ is a topological space. Tools from algebraic topology, homology in particular, are used to capture and quantify the geometry of the sub-level sets.

Recall that given any topological space $Z$, homology assigns to $Z$ a sequence of groups $H_{k}(Z), k=0,1, \ldots$ In this paper, we compute the homology groups using $\mathbb{Z}_{2}$ coefficients, and thus each $H_{k}(Z)$ is a vector space. The dimension of $H_{k}(Z)$ is called the $k$-th Betti number and is denoted by $\beta_{k}(Z)$. Betti numbers provide geometric information about $X: \beta_{0}(Z)$ is the number of connected components, or pieces, of $Z ; \beta_{1}(Z)$ indicates the number of loops or tunnels in $Z$; and $\beta_{2}(Z)$ is the number of cavities.

Our goal is to understand structure of the sub-level sets

$$
\mathrm{C}(f, \theta)=\{x \in D \mid f(x) \leq \theta\}
$$

for all values of $\theta \in \mathbb{R}$. As we vary $\theta$, the number of components, loops, and cavities in $\mathrm{C}(f, \theta)$ changes, implying that $\beta_{k}(\mathrm{C}(f, \theta)), k=0,1,2$, also changes. (See Section 4 for examples.) What is remarkable is that, under very weak conditions, we can choose bases for the vector spaces $H_{k}(\mathrm{C}(f, \theta))$ over all values of $\theta$ such that, given a basis element of $H_{k}(\mathrm{C}(f, \theta))$, we can identify a unique value $\theta_{b}$ at which this basis element appears and a unique value $\theta_{d}$ at which this basis element disappears. We refer to $\theta_{b}$ as the birth value, $\theta_{d}$ as the death value, and the pair $\left(\theta_{b}, \theta_{d}\right) \in \mathbb{R}^{2}$ as a persistence point corresponding to the chosen basis element of $H_{k}(\mathrm{C}(f, \theta))$. The difference $\theta_{d}-\theta_{b}$ is called the life span of the persistence point. Longer life spans are associated with geometric features that persist through larger variations of $\theta$, and persistence diagrams are a codification of this information. Given a scalar field $f$, the set of associated persistence diagrams are denoted by $\mathrm{PD}(f)=\left\{\mathrm{PD}_{k}(f)\right\}$, where $\mathrm{PD}_{k}(f)$ consists of all persistence points corresponding to the $k$-th level of homology. Let us stress that different features might be represented by persistence points with the same coordinates. Therefore, the persistence diagram is a multi-set, where the multiplicity of each persistence point corresponds to the number of features represented by the persistence point. Moreover, every persistence diagram contains infinitely many points at each point along the diagonal $\theta_{b}=\theta_{d}$. The reason for the inclusion of the diagonal is made clear in Definition 5.1. when we define metrics on the space of persistence diagrams.

For the systems introduced in Section 2, we first use persistent homology as a nonlinear data reduction method. For Kolmogorov flow we study the scalar field $\omega: D \rightarrow \mathbb{R}$, the $z$-component of the vorticity field, while for RayleighBénard convection we study the scalar field $T: D \rightarrow \mathbb{R}$, the temperature field at the mid-plane. It is important to note that the domains for these two scalar fields are different. For Kolmogorov flow, the domain $D$ is a torus since we are using periodic boundary conditions, while for Rayleigh-Bénard convection, $D$ is a disk. For the disk, we need only to concern ourselves with the vector spaces $H_{k}(\mathrm{C}(\omega, \theta))$ for $k=0,1$. However, for the torus, the vector 
spaces $H_{2}(\mathrm{C}(T, \theta))$ also need to be considered, since the torus encloses a three-dimensional cavity. In section 4 , we explain how the persistence diagrams $\mathrm{PD}(f)$ capture important information about the patterns given by the scalar fields $\omega$ and $T$.

The set of all persistence diagrams PD is a metric space, denoted by Per (see Section 5). Since we are studying the evolution of Kolmogorov flow and Rayleigh-Bénard convection, we have time series of the vorticity $\left\{\omega_{i}\right\}$ and temperature $\left\{T_{i}\right\}$ fields, and, therefore, we have time series of persistence diagrams $\left\{\operatorname{PD}\left(\omega_{i}\right)\right\}$ and $\left\{\operatorname{PD}\left(T_{i}\right)\right\}$. We view each of these time series as a point cloud $X \subset$ Per. To extract information about dynamical structures present in the time series, we use persistent homology a second time to quantify the geometry associated with this point cloud. This is achieved by introducing a new scalar function $f:$ Per $\rightarrow[0, \infty)$ that gives the distance from any point in Per to the point cloud $X$ and is defined by

$$
f(x):=d(x, X):=\min _{x_{i} \in X} d\left(x, x_{i}\right),
$$

where $d$ is an appropriate metric on the space of persistence diagrams. The associated sub-level sets $\mathrm{C}(f, \theta)$ are once again given by (6).

To carry out the steps mentioned above requires the ability to compute the persistence diagrams $\mathrm{PD}(f)$. To do this, we need to calculate $H_{k}(\mathrm{C}(f, \theta))$, which requires a discrete representation of $\mathrm{C}(f, \theta)$ called a complex. In the context of nonlinear data reduction, we make use of a cubical complex. When analyzing the geometry of the point cloud, we approximate $\mathrm{C}(f, \theta)$ using a Vietoris-Rips complex, which is a special form of a simplicial complex. This is a classical subject and thus there are a variety of references providing precise definitions of complexes, e.g. 7] for Vietoris-Rips complexes and 21] for cubical complexes, discussions of issues related to approximations [3], and how one proceeds from a complex to computing persistent homology [22, 7]. The homological computations in this paper were performed using the Perseus software [23].

The numerical data for the vorticity and the temperature fields is presented in the form of piecewise-constant functions defined on a rectangular lattice. For Kolmogorov flow, values of $\omega$ are reported in double precision. Recall that the vector spaces $H_{k}(\mathrm{C}(\omega, \theta))$ can only change for $\theta \in \Theta$, where $\Theta$ is the finite set of values that $\omega$ attains on the given lattice. Each of the sets $C(\omega, \theta)$ is a cubical complex, and we use the Perseus software to compute the corresponding persistence diagrams using only the values $\theta \in \Theta$. Numerical simulations for Rayleigh-Bénard convection are carried out with high precision as well. However, keeping in mind our goal to compare the numerical simulations with experimental data, we convert the temperature field to an 8-bit temperature field $T^{*}$ (an integer-valued function with values between 0 and 255), which can be obtained experimentally. Consequences of this rescaling are examined in Section 6 .

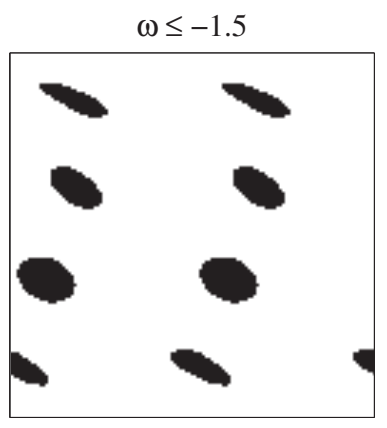

(a)

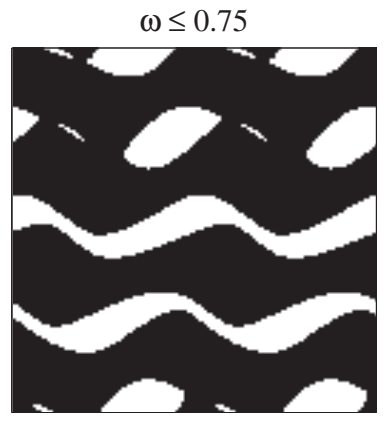

(c)

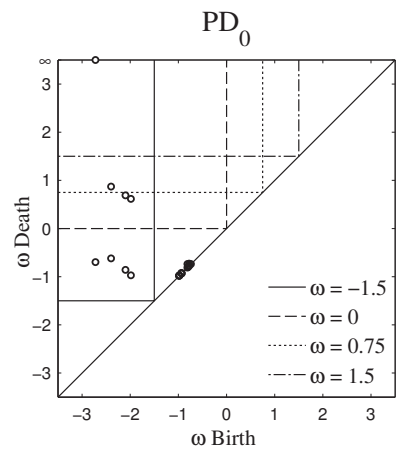

(e)

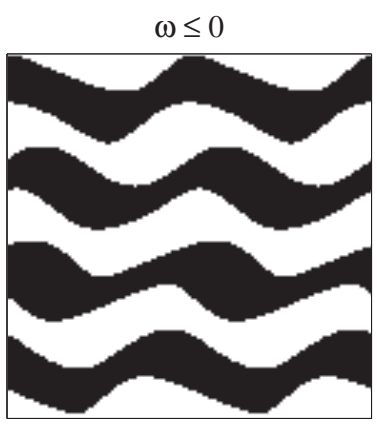

(b)

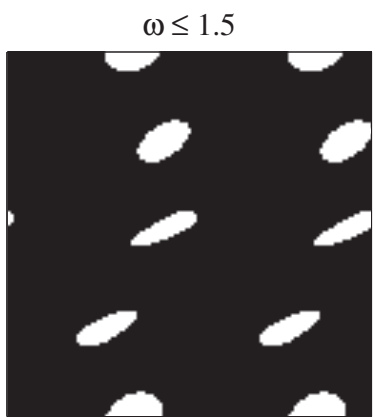

(d)

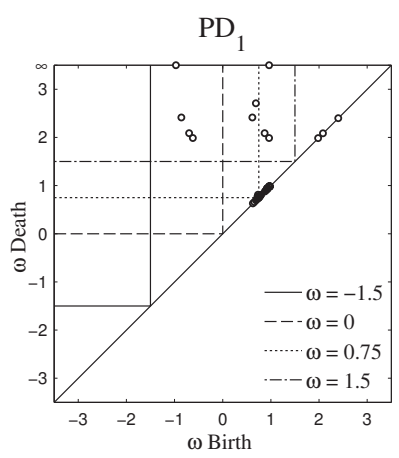

(f)
Figure 3: (a-d) Sub-level sets $\mathrm{C}(\omega, \theta)=\{x \in D: \omega(x) \leq \theta\}$ of the vorticity field, shown in Figure 2 (a), for different values of $\theta$, depicted in black. (e) $\mathrm{PD}_{0}(\omega)$ and (f) $\mathrm{PD}_{1}(\omega)$ persistence diagrams of the vorticity field indicate the values of $\theta$ at which the connected components and loops appear and disappear (merge together). Every point $\left(\theta_{b}, \theta_{d}\right)$ in the $\mathrm{PD}_{0}(\omega)\left(\mathrm{PD}_{1}(\omega)\right)$ persistence diagram corresponds to a connected component (loop) that appears in $\mathrm{C}\left(\omega, \theta_{b}\right)$ for the first time and is present in every set $\mathrm{C}(\omega, \theta)$, for $b<\theta<d$, but not in $\mathrm{C}\left(\omega, \theta_{d}\right)$. A connected component disappears by merging with a previously existing component and a loop disappears when it is filled in. Video 1 of the supplementary materials provides an animation.

\section{Interpreting Persistence Diagrams}

The purpose of this section is to provide intuition and interpretation of the information that persistence diagrams present. As indicated in the previous section, we are interested in the diagrams $\mathrm{PD}_{k}(\omega), k=0,1,2$, of the vorticity field for Kolmogorov flow, and the diagrams $\mathrm{PD}_{k}\left(T^{*}\right)$, $k=0,1$, of the temperature field for Rayleigh-Bénard con- 


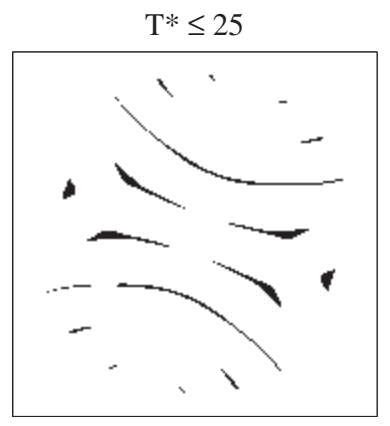

(a)

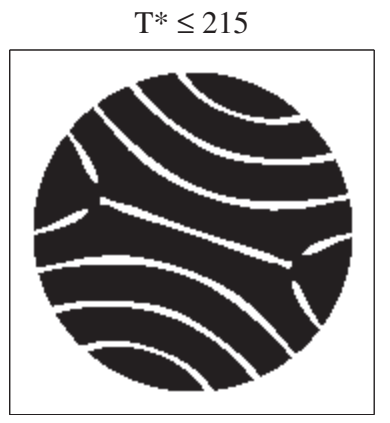

(c)

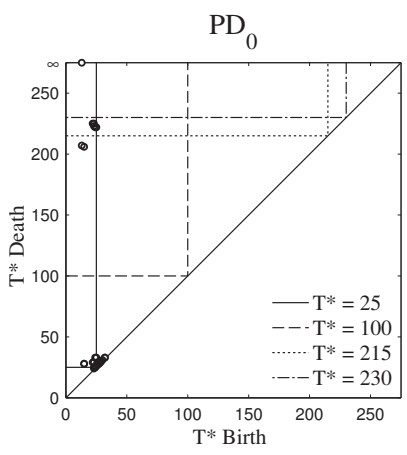

(e)

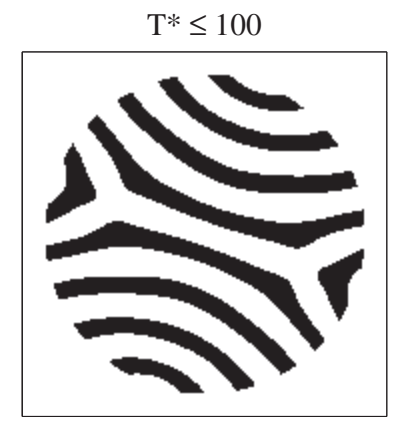

(b)

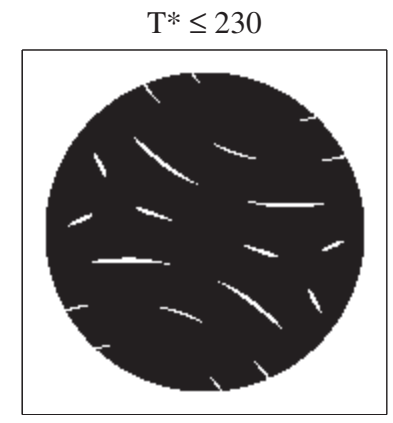

(d)

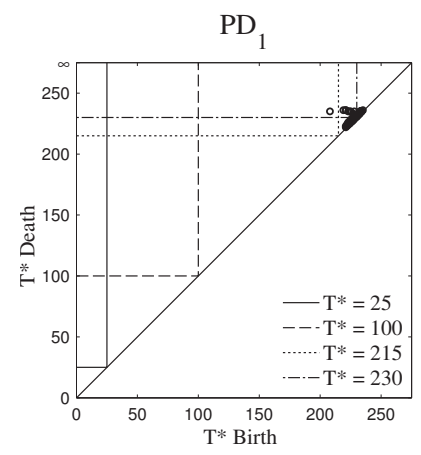

(f)
Figure 4: (a-d) Sub-level sets $\mathrm{C}\left(T^{*}, \theta\right)=\left\{x \in D: \mathbb{T}^{*}(x) \leq \theta\right\}$ of the the renormalized 8-bit temperature field $T^{*}$, shown in Figure 2(b), for different values of $\theta$, depicted in black. As before the persistence diagrams (e) $\mathrm{PD}_{0}(\omega)$ and (f) $\mathrm{PD}_{1}(\omega)$ indicate the values of $\theta$ at which the connected components and loops appear and disappear (merge together). Video 2 of the supplementary materials provides an animation.

\section{vection, shown in Figure 2}

We begin by discussing $\mathrm{PD}_{0}(\omega)$, shown in Figure 3(e), computed from a single time snapshot of the vorticity field for the Kolmogorov flow. The minimum value of the vorticity field is -2.7206 , and therefore, $\mathrm{C}(\omega, \theta)=\emptyset$ for all $\theta<-2.7206$. At $\theta=-2.7206$, two components appear, indicated by the two persistence points with birth value $\theta_{b}=-2.7206$. The death value of one of these two persistence points is $\theta_{d}=-0.697$, and so the two components merge at this value, resulting in a single component. This explains the persistence point $(-2.7206,-0.697)$. The reason the other persistence point is denoted by $(-2.7206, \infty)$, with $\theta_{d}=\infty$, is because when features merge, a choice must be made about which topological feature (in this case, a connected component) dies. Having a consistent choice of basis over all values of $\theta$ requires that the homology generator associated with the geometric feature that has the larger birth value die first. If the birth values are the same, then it does not matter which topological feature with this birth value is chosen to be the one that persists. In particular, this implies that the generator associated with one of these two initial components can never die.

Figure 3(a) indicates the subset of $D$ corresponding to $\mathrm{C}(\omega,-1.5)$. We remind the reader that the domain $D$ for Kolmogorov flow is a torus, since the left (top) and right (bottom) boundaries are identified. Therefore, $\mathrm{C}(\omega,-1.5)$ consists of eight distinct connected components instead of nine.

The existence of these eight connected components can also be extracted from $\mathrm{PD}_{0}(\omega)$, shown in Figure 3(e). Observe that these connected components correspond to connected regions with birth value $\theta_{b} \leq-1.5$ and death value $\theta_{d}>-1.5$. In Figure [3(e), this corresponds to the eight points in the rectangular region $R_{-1.5}:=$ $\left\{\left(\theta_{b}, \theta_{d}\right) \in \mathbb{R}^{2} \mid \theta_{b} \leq-1.5\right.$ and $\left.\theta_{d}>-1.5\right\}$.

Figure 3(b) indicates that $\mathrm{C}(\omega, 0)$ consists of four connected horizontal bands, which agrees with the number of persistence points in the rectangular region $R_{0}=\left\{\left(\theta_{b}, \theta_{d}\right) \in \mathbb{R}^{2} \mid \theta_{b} \leq 0\right.$ and $\left.\theta_{d}>0\right\}$ of $\mathrm{PD}_{0}(\omega)$. Each stripe is created as two distinct components present in Figure 3(a) grow and merge, causing one component to die each time. The deaths of these components are captured by the points in the rectangle $R_{-1.5}$ which are not in the rectangle $R_{0}$, since these are components that are born before $\theta=-1.5$ but die before $\theta=0$.

Three horizontal stripes merge together before $\theta=0.75$, as indicated by two points inside the rectangle $R_{-1.5}$ that are not in the rectangle $R_{0.75}$. The two remaining connected components merge together soon thereafter, and for all greater threshold values, there is only one connected component.

To finish our analysis of $\mathrm{PD}_{0}(\omega)$, we turn our attention to the persistence points close to the diagonal. These have very short life spans, which suggests that these features may be numerical artifacts. In our example, these points represent the narrow horizontal bands formed in between two connected components before they merge into a single band (see video 1 available in the supplementary materials). These narrow bands are formed by small oscillations of the vorticity field at the places where the field is almost constant.

We now turn our attention to the $\mathrm{PD}_{1}(\omega)$ persistence diagram, which characterizes loops in $\mathrm{C}(\omega, \theta)$. Appendix A provides a detailed discussion of independent loops on a torus. From $\mathrm{PD}_{1}(\omega)$, we see that the first loop appears at threshold $\theta=-0.963$. It corresponds to one of the four horizontal bands shown in Figure 3(b). Each horizontal band generates a single independent loop, corroborated by the existence of four persistence points in the rectangle 
$R_{0}$ of $\mathrm{PD}_{1}(\omega)$.

We note that the full torus has two loops captured by homology. This is expressed in $\mathrm{PD}_{1}(\omega)$ by the two persistence points with $\theta_{d}=\infty$. Observe that the first loop that appears at $\theta=-0.963$ is equivalent to one of the toral loops, thus it cannot be killed by any other loop, and hence is captured by the persistence point $(-0.963, \infty)$. The other three loops present at $\theta=0$ correspond to the same toral loop and thus must die. In fact, they do so by $\theta=2.5$. Note that the birth values $\theta_{b}$ of these persistence points are close to the death values $\theta_{d}$ of the persistence points in $R_{-1.5} \backslash R_{0}$ of $\mathrm{PD}_{0}(\omega)$. This implies that shortly after the components merge, they form horizontal bands across the entire domain.

New loops are also created as the bands start merging. If two horizontal bands are connected by $n$ links, then the number of loops generated by this object (two bands plus the links) is $(1+n)$. Thus, the first additional loop appears when a second link is created (see Appendix A). In our example, this happens near the threshold 0.75.

In Figure 3(c), there are four distinct links between the two horizontal bands at the top of the figure. The small punctures visible in Figure 3(c) are filled in almost immediately, and the four links merge into two distinct links. The points in $\mathrm{PD}_{1}(\omega)$ that are close to the diagonal capture this behavior. The other two links are present for a wider range of thresholds, and the loop they generate is represented by one of the persistence points in $\mathrm{PD}_{1}(\omega)$ with birth coordinate slightly smaller than 0.75 . The horizontal band at the top and the horizontal band at the bottom are linked in a similar fashion. This explains the presence of another point with birth coordinate slightly smaller than 0.75 .

At $\theta=0.932$, a connection from the top to the bottom boundary is created. This loop is homologically equivalent to the second of the two independent loops of the torus, and hence is identified by the persistence point $(0.932, \infty)$. As the threshold passes the value 1.988, the punctures shown in Figure 3(d) start disappearing and the corresponding loops start dying. Again, there are $1+n$ independent loops for $n>0$ punctures. Since the maximum value of $\omega$ is 2.7092, the sub-level set is the whole torus for any threshold above this, i.e. $\mathrm{C}(\omega, \theta)=D$ for all $\theta \geq 2.7092$. In this case, there are no more punctures, and the rectangle $R_{2.7092}$ contains only two persistence points.

Finally, we address the $\mathrm{PD}_{2}(\omega)$ persistence diagram, not shown for brevity. This diagram contains a single persistence point at $\left(\theta_{b}, \infty\right)=(3.01, \infty)$. The birth coordinate, $\theta_{b}$, corresponds to the minimum value of $\theta$ for which $\mathrm{C}(\omega, \theta)=D$, the whole torus. Since $\mathrm{C}(\omega, \theta)=D$ for all $\theta \geq \theta_{b}$, this point never dies.

We now discuss the persistence diagrams for the temperature field $T^{*}$ shown in Figure 4 for Rayleigh-Bénard convection. Again, beginning with $\mathrm{PD}_{0}\left(T^{*}\right)$, the points with short life spans correspond the large number of small connected components that make up $\mathrm{C}\left(T^{*}, 25\right)$, as shown in Figure 4(a). Points with long life spans represent the well-defined connected components shown in Figure 4(b). From the persistence diagram, we can see that these components merge almost simultaneously at two threshold values, $\theta \approx 210$ and $\theta \approx 225$.

Turning to $\mathrm{PD}_{1}\left(T^{*}\right)$, we note that the domain of the temperature field is a disk, so the independent loops correspond to punctures inside of the disk. The diagram $\mathrm{PD}_{1}\left(T^{*}\right)$ indicates that there are no loops with long life spans, and the loops that do appear do so roughly at the same threshold values at which the dominant components merge. These features are due to the small fluctuations of the temperature field close to the critical values at which different rolls merge together. This is consistent with their short life spans.

\section{The Space of Persistence Diagrams}

As explained in the previous section, a persistence diagram codifies, in a reasonably compact form, considerable information about the geometry of a scalar function. As suggested by the examples, we use persistence diagrams to provide a reduced description of the state of the dynamical system of interest at any given point in time. Therefore, to analyze the dynamics, we need to be able to compare one collection of persistence diagrams PD (corresponding to a snapshot of the flow pattern at an instant of time) to another collection of diagrams PD' (from another flow snapshot). There are a variety of metrics that can be imposed on persistence diagrams [24, 25, 26, 27]. The metrics used in this paper rely on pairing the points $p \in \mathrm{PD}_{k}$ in a one-to-one correspondence (bijection) with the points in $\mathrm{PD}_{k}^{\prime}$. According to the definition, every persistence diagram contains an infinite number of copies of the diagonal. Hence, there are many different bijections $\gamma$ between $\mathrm{PD}_{k}$ and $\mathrm{PD}_{k}^{\prime}$. Roughly speaking, the distance between $\mathrm{PD}$ and $\mathrm{PD}^{\prime}$ is defined using the bijections that "minimize the shift" in the mapping of the points $p$ from $\mathrm{PD}_{k}$ to $\gamma(p)$ in $\mathrm{PD}_{k}^{\prime}$. This notion is made more precise in the following definition.

Definition 5.1. Let $\mathrm{PD}=\left\{\mathrm{PD}_{k}\right\}$ and $\mathrm{PD}^{\prime}=\left\{\mathrm{PD}_{k}^{\prime}\right\}$ be two collections of persistence diagrams. The bottleneck distance between $\mathrm{PD}$ and $\mathrm{PD}^{\prime}$ is defined to be

$$
d_{B}\left(\mathrm{PD}, \mathrm{PD}^{\prime}\right)=\max _{k} \inf _{\gamma: \mathrm{PD}_{k} \rightarrow \mathrm{PD}_{k}^{\prime}} \sup _{p \in \mathrm{PD}_{k}}\|p-\gamma(p)\|_{\infty},
$$

where $\left\|\left(a_{0}, b_{0}\right)-\left(a_{1}, b_{1}\right)\right\|_{\infty}:=\max \left\{\left|a_{0}-a_{1}\right|,\left|b_{0}-b_{1}\right|\right\}$ and $\gamma$ ranges over all bijections between persistence points. Similarly, the degree-p Wasserstein distance is defined as

$$
d_{W^{p}}\left(\mathrm{PD}, \mathrm{PD}^{\prime}\right)=\left[\sum_{k} \inf _{\gamma: \mathrm{PD}_{k} \rightarrow \mathrm{PD}_{k}^{\prime}} \sum_{p \in \mathrm{PD}_{k}}\|p-\gamma(p)\|_{\infty}^{p}\right]^{1 / p} .
$$

Roughly speaking, a function $f: D \rightarrow \mathbb{R}$ is tame if, for every $\theta \in \mathbb{R}$, the vector space $H_{k}\left(f^{-1}((-\infty, \theta])\right)$ is finite 


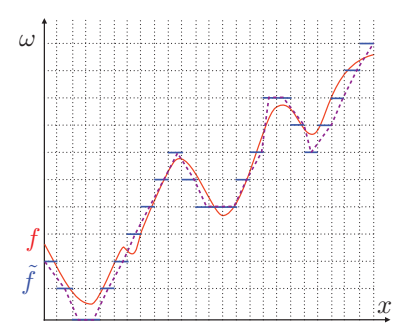

(a)

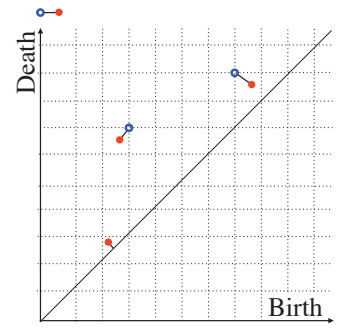

(b)
Figure 5: (a) A one-dimensional scalar field $f: D \rightarrow \mathbb{R}$ and its piecewise constant approximation $\tilde{f}$. The dashed line shows a tame approximation of $f$ whose persistence diagram is identical to $\operatorname{PD}(f)$. (b) Points in the persistence diagram $\operatorname{PD}(f)$ are given by closed symbols and the points in $\operatorname{PD}(\tilde{f})$ are represented by open symbols. Two points on the top have infinite death coordinate. Lines connecting the points represent matching of the persistence points for which the bottleneck distance $d_{B}(\operatorname{PD}(f), \operatorname{PD}(\tilde{f}))$ is realized. It follows from (10) that $d_{B}(\mathrm{PD}(f), \operatorname{PD}(\tilde{f})) \leq \sup _{x \in D}|f(x)-\tilde{f}(x)|$.

dimensional for every $k$, and there are only finitely-many thresholds at which the vector spaces change (for a precise definition see [7]). For our purposes, it suffices to remark that if $f$ is a piecewise-constant function on a finite complex, then $f$ is tame. In particular, the numericallycomputed vorticity field $\omega$ and 8-bit temperature field $T^{*}$ are tame functions.

For the remainder of this paper, we use $\mathrm{Per}_{k}$ to denote the set of persistence diagrams corresponding to $H_{k}$ and Per to denote the set of all persistence diagrams. Let $T(D, \mathbb{R})$ denote the set of tame functions $f: D \rightarrow$ $\mathbb{R}$ equipped with the $L^{\infty}$ norm. A fundamental result [7] is that, using the Wasserstein or bottleneck metrics, PD : $T(D, \mathbb{R}) \rightarrow$ Per is a Lipschitz-continuous function. In particular, if $f, g \in T(D, \mathbb{R})$, then

$$
d_{B}(\operatorname{PD}(f), \operatorname{PD}(g)) \leq \sup _{x \in D}|f(x)-g(x)| .
$$

These results on Lipschitz continuity have two important implications for this work, both stemming from the fact that our analysis is based on numerical simulations. Assume for the moment that $f: D \rightarrow \mathbb{R}$ denotes the exact solution at a given time to either Kolmogorov flow or the Boussinesq equations. Ideally, we want to understand $\mathrm{PD}(f)$. Our computations of persistent homology are based on $C\left(\tilde{f}, \theta_{i}\right)$, a cubical complex defined in terms of the numerically-reported values $\theta_{i}$, where $\tilde{f}$ represents the associated piecewise-constant function. If the numerical approximation $\tilde{f}$ satisfies $\sup _{x \in D}|f(x)-\tilde{f}(x)| \leq \varepsilon$, then by (10) we have a bound on the bottleneck distance between the actual persistence diagram $\operatorname{PD}(f)$ and the computed persistence diagram $\operatorname{PD}(\tilde{f})$, so that $d_{B}(\operatorname{PD}(f), \operatorname{PD}(\tilde{f})) \leq \varepsilon$. Figure 5 provides a schematic justification of this claim.

As indicated in the introduction, persistent homology is invariant under certain continuous deformations of the domain. To be more precise, if $g: D \rightarrow D$ is a homeomorphism and $f: D \rightarrow \mathbb{R}$, then $\operatorname{PD}(f \circ g)=\operatorname{PD}(f)$. Of particular relevance to this paper is a function $g$ which arises as a symmetric action on the domain. In this paper, we work with piecewise-constant numerical approximations of the actual functions of interest, and we cannot assume that this equality holds. However, if $f$ is given and $f^{\prime}=f \circ g$, where $g$ is as above, and we have an $L^{\infty}$ bound $\varepsilon$ on the difference between the approximation and the true function, then by (10),

$$
d_{B}\left(\mathrm{PD}(f), \operatorname{PD}\left(f^{\prime}\right)\right) \leq 2 \varepsilon .
$$

In summary, under the assumption of bounded noise or errors from numerical simulations (or experimental data), we have explicit control of the errors of the distances in Per.

\section{Using Metrics in the Space of Persistence Dia- grams}

The goal of this section is twofold: one, to provide intuition about the information contained in the different metrics, and two, to suggest how viewing a time series in Per can provide insight into the underlying dynamics.

We begin by remarking that the bottleneck distance $d_{B}$ measures only the single largest difference between the persistence diagrams and ignores the rest. The Wasserstein distance $d_{W^{p}}$ includes all differences between the diagrams. Thus, it is always true that

$$
d_{B} \leq d_{W^{p}} .
$$

The sensitivity of the Wasserstein metric to small differences (possibly due to noise) can be modulated by the choice of the value of $p$, i.e. if $p>q$, then one expects $d_{W^{p}}$ to be less sensitive to small changes than $d_{W^{q}}$. In this paper, we restrict ourselves to the bottleneck distance $d_{B}$ and the Wasserstein distances $d_{W^{p}}$ for $p=1,2$.

The most obvious use of these metrics is to identify or distinguish patterns. As an example, we consider patterns along an orbit from the Kolmogorov flow. As indicated in Section 2.1. this particular trajectory arises from a periodic orbit with a slow drift along an orbit of continuous symmetry. In particular, we consider the three time points indicated in Figure 1(a): two that appear to differ by the continuous symmetry, and a third that lies on the "opposite' side of the periodic orbit. Plots of the associated vorticity fields at these points (see Figure 6) agree with this characterization of the time points. We want to identify this information through the associated persistence diagrams $\mathrm{PD}^{a}, \mathrm{PD}^{b}$, and $\mathrm{PD}^{c}$, shown in Figure 7. Indeed, the plots of $\mathrm{PD}_{k}^{a}$ and $\mathrm{PD}_{k}^{b}$ are difficult to distinguish, but $\mathrm{PD}_{k}^{c}$ is clearly distinct. To quantify this difference, we make use of the distances between the persistence diagrams using $d_{B}, d_{W^{2}}$, and $d_{W^{1}}$. These values are recorded in Table 1 Not surprisingly, the distances between $\mathrm{PD}^{a}$ and $\mathrm{PD}^{b}$ are much smaller than the distances between $\mathrm{PD}^{a}$ and $\mathrm{PD}^{c}$. We want to use these distances, as opposed to the detailed information in the persistence diagrams, to obtain rough 


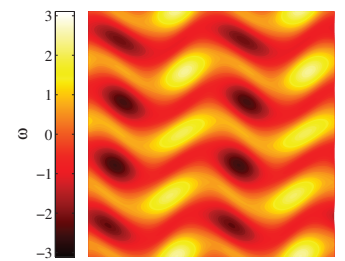

(a)

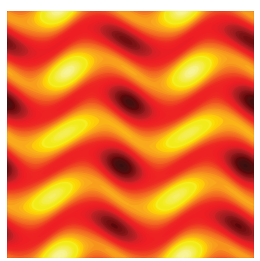

(b)

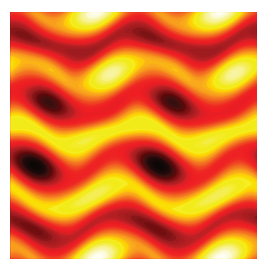

(c)
Figure 6: Three snapshots of the vorticity fields $\omega$ from the stable relative periodic orbit of the Kolmogorov flow, found at $R e=25.43$. The vorticity fields correspond to the (a) diamond, (b) square, and (c) circle in Figure 1a). The persistence diagrams for these three snapshots are generated and compared in Figure 7 Differences between the persistence diagrams are qualitatively measured by the distances shown in Table 1
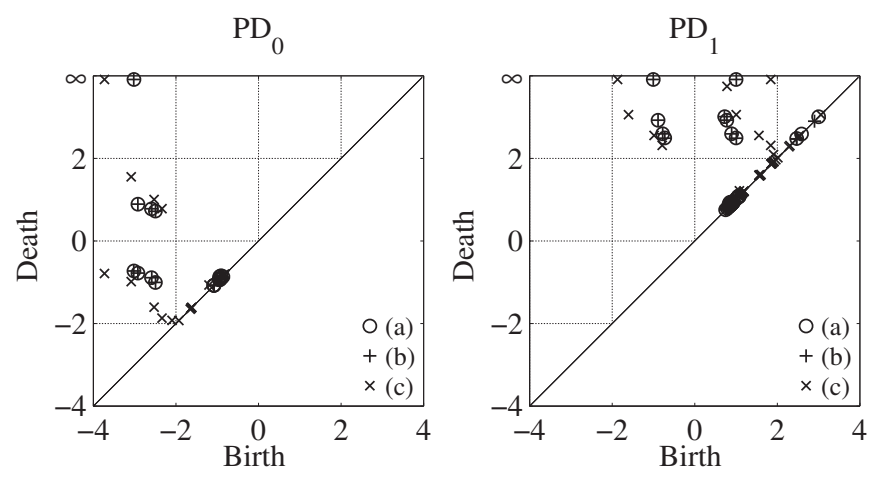

Figure 7: $\mathrm{PD}_{0}$ persistence diagrams $\mathrm{PD}^{a}, \mathrm{PD}^{b}$ and $\mathrm{PD}^{c}$ for the vorticity fields shown in Figure 6 The points in $\mathrm{PD}^{a}$ and $\mathrm{PD}^{b}$ are almost identical because the corresponding vorticity fields are similar. The points in $\mathrm{PD}^{c}$ are more spread out and do not shadow the points in $\mathrm{PD}^{a}$ so well. The same is true for the $\mathrm{PD}_{1}$ persistence diagrams which are not shown. So $d_{\star}\left(\mathrm{PD}^{a}, \mathrm{PD}^{b}\right)<d_{\star}\left(\mathrm{PD}^{a}, \mathrm{PD}^{c}\right)$, for $\star \in\left\{B, W^{2}, W^{1}\right\}$, as indicated by Table 1

information about how the pattern at Figure 6(a) differs from the pattern at Figure 6(c).

The patterns shown in Figure 6(a)-(b) differ by a symmetric transformation, and so $d_{B}\left(\mathrm{PD}^{a}, \mathrm{PD}^{b}\right)$ can be interpreted as a lower bound on the numerical errors. Now observe that either $\mathrm{PD}^{a}$ or $\mathrm{PD}^{c}$ must have a persistence point with life span greater than $d_{B}\left(\mathrm{PD}^{a}, \mathrm{PD}^{c}\right)$ (otherwise pairing the persistence points with the diagonal will produce a smaller $d_{B}$ distance). Since the ratio $d_{B}\left(\mathrm{PD}^{a}, \mathrm{PD}^{c}\right) / d_{B}\left(\mathrm{PD}^{a}, \mathrm{PD}^{b}\right)$ is 86.4 , we know that there is a significant distinction between differences that should be ascribed to error and differences that can be ascribed to significant geometric features.

\begin{tabular}{c|ccc}
\hline & $d_{B}$ & $d_{W^{2}}$ & $d_{W^{1}}$ \\
\hline$\left(\mathrm{PD}^{a}, \mathrm{PD}^{b}\right)$ & 0.01 & 0.049 & 0.497 \\
$\left(\mathrm{PD}^{a}, \mathrm{PD}^{c}\right)$ & 0.864 & 2.648 & 12.35 \\
ratio $\frac{\left(\mathrm{PD}^{a}, \mathrm{PD}^{c}\right)}{\left(\mathrm{PD}^{a}, \mathrm{PD}^{b}\right)}$ & 86.4 & 54.05 & 24.85
\end{tabular}

Table 1: Distances between selected persistence diagrams (rounded to 3 decimal places) shown in Figure 7 corresponding to the vorticity fields given by Figure 6

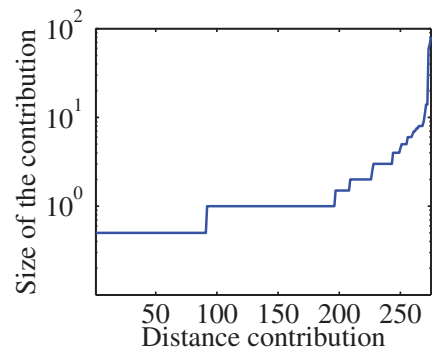

(a)

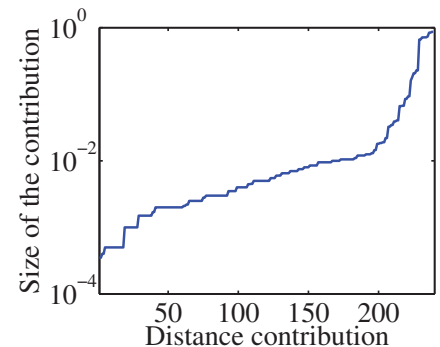

(b)
Figure 8: (a)Contributions to the $d_{W^{1}}\left(\mathrm{PD}^{A}, \mathrm{PD}^{C}\right)$ distance for Rayleigh-Bénard convection. The sizes of the contributions are given by the distances $\|p-\gamma(p)\|_{\infty}$ between the points paired together by the minimal matching $\gamma$. All of these distances contribute to $d_{W^{1}}$ distance, which is obtained by summing up the individual contributions. (b) Contributions to the $d_{W^{1}}\left(\mathrm{PD}^{a}, \mathrm{PD}^{c}\right)$ distance for the Kolmogorov flow.

For some applications, there might be only two different scales at which the geometric features evolve: one scale corresponding to the signal, and the other representing the noise fluctuations. If that is the case, then there are only two types of changes. If we suppose that the large changes are comparable to $d_{B}\left(\mathrm{PD}^{a}, \mathrm{PD}^{c}\right)$ and the noise fluctuations are of the order $d_{B}\left(\mathrm{PD}^{a}, \mathrm{PD}^{b}\right)$, then we can approximate the distances $d_{W^{1}}\left(\mathrm{PD}^{a}, \mathrm{PD}^{c}\right)$ and $d_{W^{2}}\left(\mathrm{PD}^{a}, \mathrm{PD}^{c}\right)$ as follows:

$$
\begin{aligned}
& d_{W^{1}}\left(\mathrm{PD}^{a}, \mathrm{PD}^{c}\right) \approx n \cdot d_{B}\left(\mathrm{PD}^{a}, \mathrm{PD}^{c}\right)+k \cdot d_{B}\left(\mathrm{PD}^{a}, \mathrm{PD}^{b}\right), \\
& d_{W^{2}}\left(\mathrm{PD}^{a}, \mathrm{PD}^{c}\right) \approx \\
& \sqrt{n\left(d_{B}\left(\mathrm{PD}^{a}, \mathrm{PD}^{c}\right)\right)^{2}+k\left(d_{B}\left(\mathrm{PD}^{a}, \mathrm{PD}^{b}\right)\right)^{2}}
\end{aligned}
$$

where $n$ is the number of features that change significantly and $k$ is the number of features that change very little. We recall that $d_{B}\left(\mathrm{PD}^{a}, \mathrm{PD}^{b}\right) \ll d_{B}\left(\mathrm{PD}^{a}, \mathrm{PD}^{c}\right)$ (Table 1). Hence, the significant contributions to the $d_{W^{2}}$ metric are of the order of $d_{B}\left(\mathrm{PD}^{a}, \mathrm{PD}^{c}\right)$, and the small changes do not significantly contribute to $d_{W^{2}}$. This leads to the following approximation:

$$
d_{W^{2}}\left(\mathrm{PD}^{a}, \mathrm{PD}^{c}\right) \approx \sqrt{n\left(d_{B}\left(\mathrm{PD}^{a}, \mathrm{PD}^{c}\right)\right)^{2}} .
$$

By solving (13) and (15), we obtain $n=9$ and $k=383$, and so the number of features that change significantly is bounded from below by 9 . Before we discuss the values of $n$ and $k$, let us repeat the same computation for the Rayleigh-Bénard convection.

We recall that the 8 -bit temperature field $T^{*}$ is an integer-valued function with values between 0 and 255 . For integer-valued functions, the smallest nonzero $d_{B}$ distance between distinct frames is $e=0.5$. We use this number as the lower bound on the numerical errors. The snapshots $A$ and $C$, not shown for brevity, are from a single orbit, and they realize the maximal distance between two snapshots (exact distances are given by Table 21). Solving Equations (13) and (15) yields $n=35$ and $k=-4400$. These numbers obviously do not make sense. Therefore, the changes 


\begin{tabular}{c|ccc}
\hline & $d_{B}$ & $d_{W^{2}}$ & $d_{W^{1}}$ \\
\hline$\left(\mathrm{PD}^{A}, \mathrm{PD}^{C}\right)$ & 81.5 & 480.9 & 650.5
\end{tabular}

Table 2: Distances between selected persistence diagrams (rounded to 3 decimal places) corresponding to two different 8-bit temperature fields obtained from a single orbit of Rayleigh-Bénard convection.

cannot be divided into two distinct groups, as we assumed above. Figure 8 (a) shows that there is only one change on the order of $d_{B}\left(\mathrm{PD}^{A}, \mathrm{PD}^{C}\right)$. All the other changes are at least an order of magnitude smaller. More precisely, there are 77 changes that are between one to two orders of magnitude smaller than $d_{B}\left(\mathrm{PD}^{A}, \mathrm{PD}^{C}\right)$. Moreover, these changes are at least an order of magnitude larger than our error estimate $e=0.5$, and there are also 105 changes of the size $2 e$. Due to the significant number of contributions at all orders of magnitude between the noise estimate and $d_{B}\left(\mathrm{PD}^{A}, \mathrm{PD}^{C}\right)$, we cannot assume that there are predominantly two distinct types of changes: one corresponding to the noise and the other to the signal. Thus, the approximation (15) of $d_{W^{2}}$ is not valid in this setting because a large part of $d_{W^{2}}\left(\mathrm{PD}^{A}, \mathrm{PD}^{C}\right)$ comes from contributions at the intermediate scales between $e$ and $d_{B}\left(\mathrm{PD}^{A}, \mathrm{PD}^{B}\right)$.

We now return to the values of $n$ and $k$ for the Kolmogorov flow. Figure 8(b) shows that there are approximately 200 changes of order smaller or equal to $d_{B}\left(\mathrm{PD}^{a}, \mathrm{PD}^{b}\right)$, and 11 dominant changes of order $d_{B}\left(\mathrm{PD}^{a}, \mathrm{PD}^{c}\right)$. Finally, we can identify approximately 28 changes occurring on intermediate scales, and their sizes are at least an order of magnitude smaller than $d_{B}\left(\mathrm{PD}^{a}, \mathrm{PD}^{c}\right)$. In fact, most of them are not much larger than $d_{B}\left(\mathrm{PD}^{a}, \mathrm{PD}^{b}\right)$. Hence, the changes can be roughly divided into two classes of different order. The fact that the division is not absolutely sharp leads to $n=9$, which is smaller than the actual number of dominant changes on the order of $d_{B}\left(\mathrm{PD}^{a}, \mathrm{PD}^{c}\right)$.

We now turn to the question of understanding dynamics from the time series in Per. Let $f_{i}$ denote the scalar field of the system at time $t_{i}$. If $\Delta t=t_{i+1}-t_{i}$ is small and the evolution of the system is continuous, then because $d_{\star}$ (for $\left.\star \in\left\{B, W^{2}, W^{1}\right\}\right)$ is a metric,

$$
s_{\star}\left(t_{i}\right)=\frac{d_{\star}\left(\mathrm{PD}\left(f_{i}\right), \mathrm{PD}\left(f_{i+1}\right)\right)}{\Delta t}
$$

can be interpreted as an average speed in the space of persistence diagrams over the time interval $\left[t_{i}, t_{i+1}\right]$. The value of $s_{\star}$ depends on the choice of metric. For example, $s_{d_{B}}$ is the rate at which the largest change between the geometric features of the scalar fields occurs. The speeds measured by $d_{W^{p}}, p=1,2$, keep track of the rate of change between all geometric features, though to some extent, $d_{W^{2}}$ suppresses the effect of noise.

Figure 9(a) shows distances $d_{*}$ between consecutive sample points, normalized by the maximum distance, of samples taken along approximately three periods of the stable relative periodic orbit of the Kolmogorov flow described in Section 2.1. Normalizing $s_{*}$ by the maximum speed along

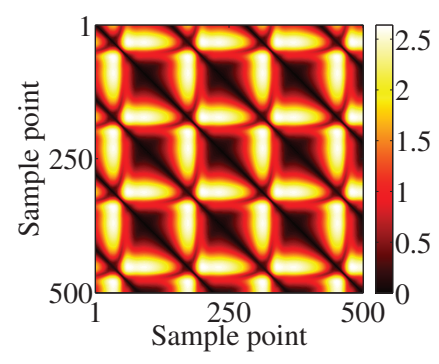

(a)

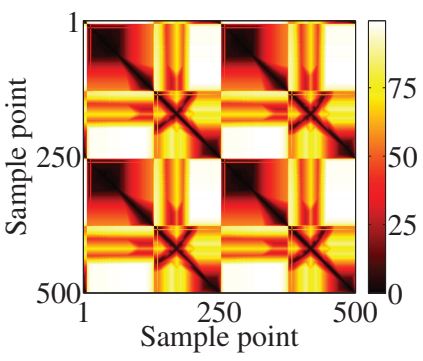

(b)
Figure 10: (a) Distance matrix $D$, generated by the $d_{W^{2}}$ metric, for approximately three periods of the stable relative periodic orbit of the Kolmogorov flow. The large black patches correspond to the parts of the orbit with slow dynamics. Equally spaced black lines parallel to the diagonal suggest periodicity of the orbit with period equal to the distance between these lines. (b) Distance matrix $D$, generated by the $d_{B}$ metric, for 2 periods of the almost-periodic orbit of Rayleigh-Bénard convection. The checkerboard pattern indicates that sampling is too sparse, and fast dynamics are not resolved with the level of sampling.

the orbit furnishes the same curves. Each of the graphs of $s_{\star}$ indicate that speed is not uniform along the orbit; there are parts of the orbit where the geometry is changing slowly, separated by intervals of relatively fast evolution. The evolution is extremely slow around the states 100, 240, and 380 . The values of the speed (before normalizing) are below the noise (fluctuation) levels given by the first row of Table 1. This suggests that the orbit may be passing close to a fixed point.

While the general shapes of the speed profiles for different distances are similar, there are places where the signs of their derivatives differ. As the system starts accelerating around $t=100$, all three speeds are increasing. Around $t=130$, the speed $s_{W^{1}}$ starts decreasing while the other two speeds are still increasing. Note that around $t=130$, the speeds rise above the noise level (fluctuations). The fact that $s_{B}$ and $s_{W^{2}}$ are both increasing means that the changes between the prominent geometric features are growing in this region. The speed $s_{W^{1}}$ is decreasing in this region and so the noise (error) fluctuations are decreasing. At $t=170$, the dominant geometric features start to evolve considerably. Changes of the dominant features are the most important contributions to all three metrics. Therefore, the derivatives of the speeds $s_{\star}$ have the same sign again (see video 3,4 , or 5 in the supplementary materials).

Figure 9(b) shows the normalized speed profiles for the Rayleigh-Bénard convection simulations. As in the case of the Kolmogorov flow, all three metrics indicate that there are two distinct speed scales along the orbit. However, the speed profile for $d_{B}$ differs significantly from those of $d_{W^{1}}$ and $d_{W^{2}}$. In particular, it suggests that for significant time periods, the major geometric features of the temperature field vary only slightly, followed by two rapid bursts of change. This can be verified by viewing video 6,7 , or 8 of the supplementary materials. Away from the rapid bursts, 


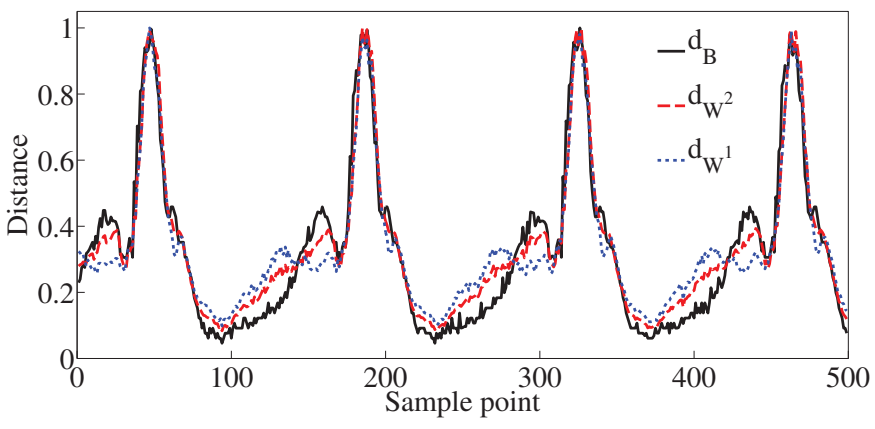

(a)

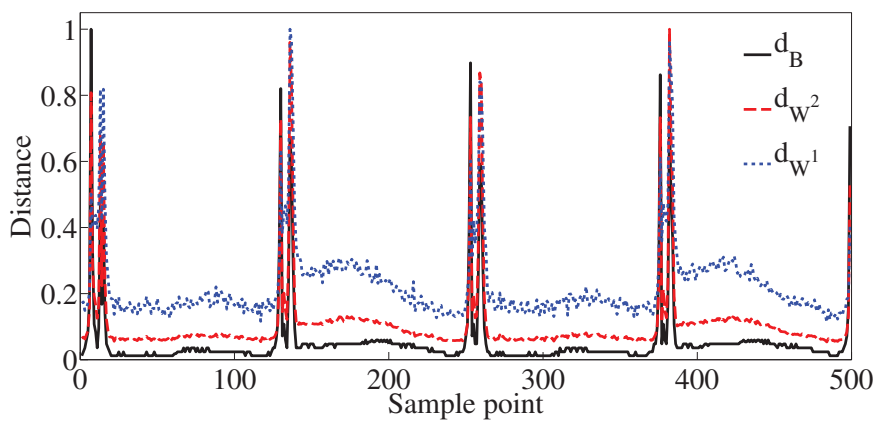

(b)

Figure 9: (a) Distances $d_{\star}$ between the consecutive sample points along the stable relative periodic orbit of the Kolmogorov flow are shown for just over three periods. Distances are normalized by their maximum value which is 0.0654 for $d_{B}, 0.2266$ for $d_{W^{2}}$, and 1.9143 for $d_{W^{1}}$. Distance between the consecutive sample points can be interpreted as the speed at which the orbit is traversed in the space of persistence diagrams. Speed is not uniform along the orbit. Instead, there are parts of the orbit where the dynamics are slow, separated by relatively fast evolution. (b) Distances $d_{\star}$ between the consecutive sample points along an almost-periodic orbit from Rayleigh-Bénard convection are shown for approximately 2 periods. Distances are again normalized by their maximum value which is 83.5 for $d_{B}, 113.66$ for $d_{W} 2$, and 383 for $d_{W^{1}}$.

$s_{B}$ is close to 1 . The temperature field has integer values, so the changes cannot be smaller than 0.5. This implies that both $s_{W^{1}}$ and $s_{W^{2}}$ are dominated by the small fluctuations which are roughly of order 1 . Hence, the relative speeds $s_{W^{1}}$ and $s_{W^{2}}$ have essentially the same shape.

The plots of $s_{\star}$ hint at the underlying dynamics being that of a periodic orbit. However, it is important to keep in mind that Per is an infinite-dimensional space, and thus periodicity in the speed of a trajectory does not imply that the trajectory lies on a closed curve. As an example, Figure 9(b) suggests that there are just over four periods of Rayleigh-Bénard convection shown, and that a single period is roughly 125 frames long. However, looking at video 6,7 , or 8 (in the supplementary materials), it is clear that a full period is closer to 250 frames. Similarly, it is not obvious that extended periods of high speed imply that the pattern changes significantly over that time period (a periodic orbit of small diameter can exhibit high speed). This requires a more global geometric analysis of the time series, which we discuss shortly.

With the same data set used to generate Figure 9(a) and letting $\omega_{j}$ denote the vorticity field at time $t_{j}$, Figure 10(a) exhibits the $d_{W^{2}}$ distance matrix $D$ for Kolmogorov flow, with color-coded entries $D(i, j):=d_{W^{2}}\left(\operatorname{PD}\left(\omega_{i}\right), \operatorname{PD}\left(\omega_{j}\right)\right)$. (The $d_{B}$ and $d_{W^{1}}$ distance matrices look very similar and are not shown.) Observe that $D(i, i)=0$ and $D$ is symmetric since $D(i, j)=D(j, i)$. Furthermore, Figure 9(a) is a plot of the immediate off-diagonal entries. A striking feature of the distance matrix in Figure 10(a) is the existence of dark lines parallel to the diagonal, spaced at intervals of roughly 110 samples. This indicates that, in the space of persistence diagrams, the trajectory periodically repeats the same, or nearly the same, state. Since the diagonals are spaced at roughly 110 samples, we can indeed say that the orbit revisits very similar states at intervals of roughly 110 samples. Similarly, the light regions close to the diagonal in Figure 10(a) correspond to the times in Figure 9(a) at which the speed is large, indicating significant changes in the pattern at these times.

To obtain a more global analysis we turn to Figure 10(b) that shows the distance matrix $D(i, j):=$ $d_{W^{2}}\left(\operatorname{PD}\left(T_{i}^{*}\right), \operatorname{PD}\left(T_{j}^{*}\right)\right)$ for the temperature fields $T_{i}^{*}$ corresponding to the trajectory from Rayleigh-Bénard convection. Distances between the consecutive temperature fields are shown in Figure 9(b). The dark diagonal lines are spaced at intervals of roughly 250 samples. Thus, even though the Figure 9(b) suggests a period of approximately 125 , the orbit does not revisit the same state in the space of persistence diagrams every 125 samples, but instead every 250 samples.

\section{Analyzing a Point Cloud using Persistent Ho- mology}

The discussion in the previous section suggests that interesting information concerning the dynamics of the geometry of time-evolving scalar fields can be obtained by studying the time series in the space of persistence diagrams. Note that each scalar field is represented by a persistence diagram $\operatorname{PD}(f)$ and thus corresponds to a point in Per. We argue that viewing the time series as a point cloud in the space of persistence diagrams and studying its geometry provides useful information about the dynamics.

For a point cloud $X \subset$ Per and the scalar function $f: X \rightarrow[0, \infty)$ given by (7) (for any of the metrics $d_{B}, d_{W^{2}}$ or $\left.d_{W^{1}}\right)$, the sub-level set $\mathrm{C}(f, \theta)$ defined by (6) is a union of balls

$$
\mathrm{C}(f, \theta)=\bigcup_{\mathrm{PD} \in X} B(\mathrm{PD}, \theta),
$$

where $B(\mathrm{PD}, \theta)=\left\{\mathrm{PD}^{\prime} \in \operatorname{Per} \mid d\left(\mathrm{PD}^{\prime}, \mathrm{PD}\right) \leq \theta\right\}$, and $d$ is the appropriate metric. In general, one should expect that the sets $\mathrm{C}(f, \theta)$ are complicated. Therefore, computing $H_{*}(\mathrm{C}(f, \theta))$ directly is not practical. Instead, we make use of the following complex. 


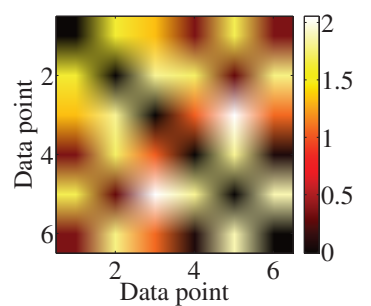

(a)

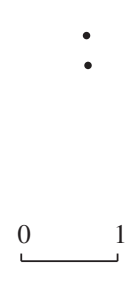

(b)

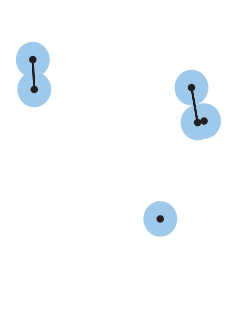

(c)

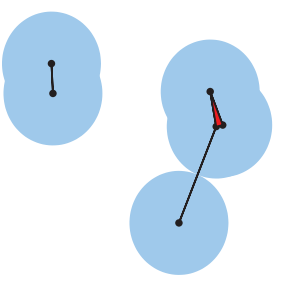

(d)

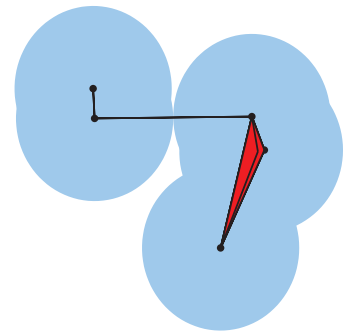

(e)

Figure 11: (a) Distance matrix representing pairwise Euclidian distances $d_{E}$ between the points in (b) a point cloud $X$. (c-e) The blue shaded regions indicate the sub-level sets $\mathrm{C}(X, \theta)$ for $\theta=0,0.1755,0.5135$, and 0.816 . The points, edges and triangles indicate the Vietoris-Rips complexes $\mathrm{R}(X, \theta)$. (c) For $\theta=0.1775$ the set $\mathrm{C}(f, \theta)$ consist of three distinct connected clusters. The same is true for $\mathcal{R}(X, \theta)$. The points in each connected component of $\mathrm{C}(f, \theta)$ are connected by edges in $\mathcal{R}(X, \theta)$. (c) The three components remain distinct until $\theta=0.5135$, at which point two components of $\mathrm{C}(f, \theta)$ merge and an edge connecting the points in the merged components appears in $\mathcal{R}(X, \theta)$.

Definition 7.1. Given a point cloud $X=\left\{x_{0}, \ldots, x_{N}\right\}$ in a metric space with distance function $d$, the VietorisRips complex at scale $\theta$, denoted $\mathrm{R}(X, \theta)$, is the simplicial complex defined by the collection of simplicies

$\left\{\left\langle x_{n_{0}}, \ldots, x_{n_{k}}\right\rangle \mid d\left(x_{n_{i}}, x_{n_{j}}\right) \leq 2 \theta\right.$, for all $\left.i, j \in\{0,1,2, \ldots, k\}\right\}$.

Observe that the Vietoris-Rips complex is determined by the distance matrix associated with $X$, and hence, there is a finite set of threshold values $\Theta=\left\{\theta_{i}\right\}$ at which the complex changes. Thus, given a point cloud $X$ in a metric space with metric $d$, the associated persistence diagrams $\operatorname{PD}(X, d)$ are determined by the Vietoris-Rips complexes $\mathrm{R}(X, \theta)$ for $\theta \in \Theta$.

We emphasize that the only data used to analyze a point cloud based on the persistent homology of Vietoris-Rips complexes are the pairwise distances between the points given by the distance matrix associated with $X$.

\subsection{Detecting Clusters}

Since $\beta_{0}$ counts components, it is reasonable to use persistent homology as a clustering tool. We demonstrate this idea on a point cloud with pairwise distances given by the distance matrix shown in Figure 11(a). A possible configuration of the six points in $\mathbb{R}^{2}$ is depicted in Figure 11(b). Using the length scale presented in Figure 11(b) as an indicator of the order of magnitude at which we want to declare a separation length for the clusters, there are three clusters. We now focus on the geometric information conveyed by $\mathrm{PD}_{0}\left(X, d_{E}\right)$, shown in Figure 12 .

Observe that $\mathrm{C}(f, 0)=\mathrm{R}(X, 0)$ consists of 6 vertices. As $\theta$ increases, the distinct connected components of $\mathrm{C}(f, \theta)$ (as defined in (17)) start merging together. In fact, when the balls $B\left(x_{i}, \theta\right)$ and $B\left(x_{j}, \theta\right)$ merge together, an edge $\left\langle x_{i}, x_{j}\right\rangle$ appears in $\mathrm{R}(X, \theta)$. Therefore, $H_{0}(\mathrm{C}(f, \theta))=$ $H_{0}(\mathrm{R}(X, \theta))$ for all $\theta \in \mathbb{R}$, and $\mathrm{PD}_{0}(f)=\mathrm{PD}_{0}\left(X, d_{E}\right)$. Note that it is impossible for a new connected component to appear for $\theta>0$. Hence, all persistence points in $\mathrm{PD}_{0}\left(X, d_{E}\right)$ have a birth value equal to zero. The death coordinates represent the spatial scales at which distinct

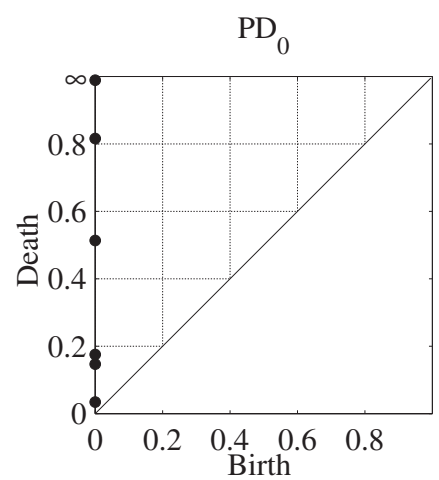

Figure 12: Persistence diagram $\mathrm{PD}_{0}\left(X, d_{E}\right)$ corresponding to the distance matrix in Figure 11(a).

connected components (clusters) merge together. Say that we are interested in clusters where the minimal separation is on the order of 1 length scale. These clusters correspond to the points in $\mathrm{PD}_{0}\left(X, d_{E}\right)$ with the death coordinate greater than approximately 0.5 , and there are three persistence points that satisfy this criterion. Thus, we declare that there are three clusters. If the relevant scale for separation is of an order of magnitude smaller, then there are five clusters, since, in addition to the three points with death value greater than 0.5 , two points have death values slightly larger than 0.05 .

Alternatively, if we are interested in dividing the data into two clusters, then $\mathrm{PD}_{0}\left(X, d_{E}\right)$ can be used to determine the magnitude of the separation between the clusters. Observe that the persistence point $(0, \infty)$ corresponds to the final connected component. The persistence point $(0,0.816)$, with the largest finite death coordinate, indicates that the components merged at a distance 0.816 . Hence, the minimal distance between points from the point cloud $X$ that belong to two distinct clusters is 1.632 .

\subsection{Detecting Loops}

Since $\beta_{1}$ counts loops, it is reasonable to use persistent homology as a tool for identifying cycles that arise 


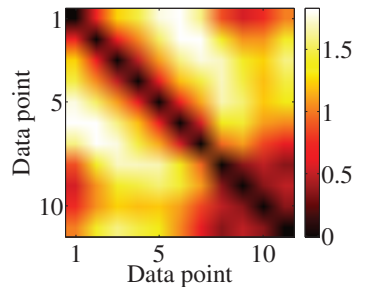

(a)

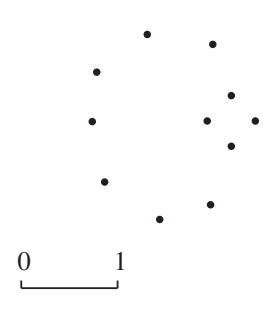

(b)

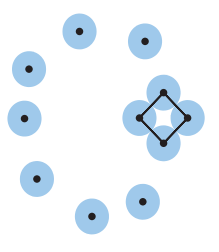

(c)

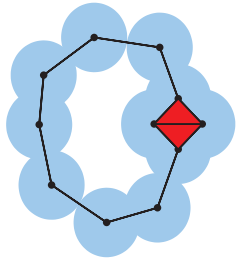

(d)

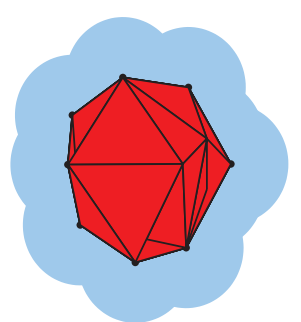

(e)

Figure 13: (a) Distance matrix representing pairwise Euclidian distances $d_{E}$ between the points in (b) a point cloud $X$. (c-e) Sets C $(f, \theta)$ for $\theta=0,0.177,0.343$ and 0.596 . The homology of $\mathrm{C}(f, \theta)$ can be approximated by a Vietoris-Rips complex $\mathrm{R}(X, \theta)$ given by the vertices, edges, and triangles shown in (b-e). The first loop in $\mathrm{C}(f, \theta)$ is created at $\theta=0.177$. This loop is due to the noisy sampling and is filled in almost immediately. The dominant loop shown in (c) is formed at $\theta=0.343$ and persists until $\theta=0.596$.

from dynamics. Consider any point cloud that generates a distance matrix as in Figure 13(a). Again, for the sake of intuition, we present in Figure 13(b) an example of a point cloud $X \subset \mathbb{R}^{2}$ with pairwise distances given by the distance matrix shown. The persistence diagrams for the associated Vietoris-Rips complex filtrations are shown in Figure 14.

Applying the reasoning from the previous section, we can ask whether there is a natural or interesting clustering of the data. If, as before, we insist that we are interested in clusters where the minimal separation is on the order of length scale 1, shown in Figure 13(b), then $(0, \infty)$ is the only persistence point with death value greater than 0.5 , i.e. at this scale there is only one component. Thus, we conclude that from a geometric perspective we may treat the point cloud as arising from a single dynamical structure.

We now look for cyclic structures. Observe that $\mathrm{PD}_{1}\left(X, d_{E}\right)$ contains two persistence points. The life span of point $(0.177,0.250)$ is 0.06 , which is short compared to the order 1 length scale, and thus it is reasonable to think of this as a result of noise in the data. This is substantiated by Figure 13(c), in which the loop in the VietorisRips complex $\mathrm{R}(X, 0.177)$ consists of four edges. An additional edge and two triangles (two 2-simplicies) appear in $\mathrm{R}(X, 0.250)$, see Figure 13(d). The triangles fill in the loop formed by the edges of $\mathrm{R}(X, 0.177)$. Two of the four data points that are involved in the construction of this loop can be viewed as arising from noise or errors associated with sampling points from a smooth cycle.

The life span of persistence point $(0.343,0.596)$ is 0.253 and suggests that the point cloud is generated by a loop with a minimal radius of 0.596 , which is on the order of the scale of the data. This suggests that the associated cycle, indicated in Figure 13(d), represents an observable, robust dynamical feature.

\subsection{Application to Systems with Multiple Time Scales and Large Data Sets}

Characterizing the geometry of a continuous orbit via an approximation by a discrete time series depends on the

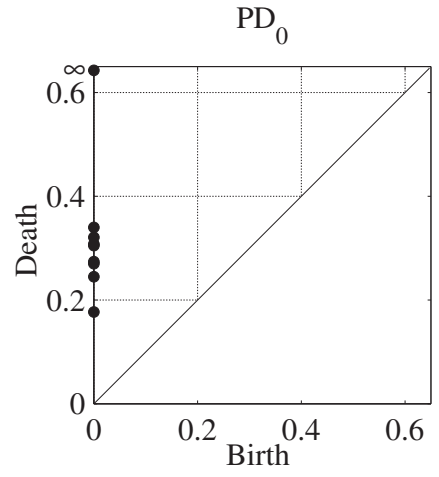

(a)

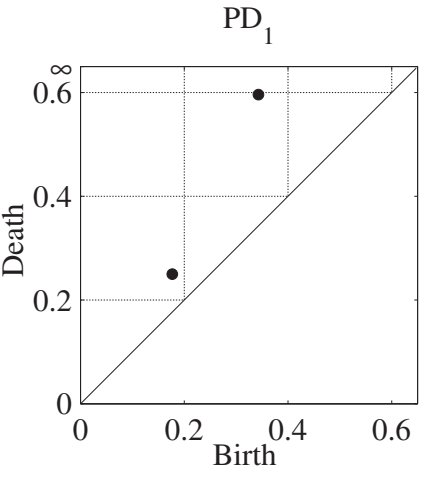

(b)
Figure 14: Persistence diagrams (a) $\mathrm{PD}_{0}\left(X, d_{E}\right)$ and (b) $\mathrm{PD}_{1}\left(X, d_{E}\right)$ corresponding to the distance matrix in Figure 13 (a). The persistence diagram $\mathrm{PD}_{1}\left(X, d_{E}\right)$ contains a dominant point $(0.343,0.596)$ corresponding to the robust loop shown in Figure 13(d) while the point $(0.177,0.25)$ represents the small loop visible in Figure 13 (c).

frequency of sampling, and thus becomes a challenge in the setting of dynamics with multiple time scales, i.e. when the rate of change of the patterns is far from constant. If the sampling rate is too slow, then parts of the orbit will be poorly (or not at all) sampled. Note that the geometry of the continuous trajectory may be more complicated than that of a circle; secondary structures might occur if the orbit is twisted, pinched, or bent in Per. Thus, the missing parts of the orbit could distort (or entirely miss) significant features in the geometry of the sampled trajectory as compared to the geometry of the underlying (continuous) dynamics. Thus, in order to obtain a description of the geometry on all relevant spatial scales, including information about secondary structures, the sampling rate needs to be fast enough.

To determine if a trajectory has been sampled densely enough to resolve the geometry of the underlying dynamics, it is useful to compare the following three values related to the point cloud in Per: the noise threshold of the system, the maximum consecutive distance in the sampled trajectory, and the diameter of the point cloud. Ide- 
ally, once a noise threshold has been computed, one would like distances between consecutive points from the sampled trajectory to be on the length scale of the noise. If sampling faster than this, the features detected from the sample that are on the scale of the noise would be indistinguishable from artifacts generated from the noise in the sample. Thus, ideally, the distance profiles (e.g. Figure 9 for Kolmogorov flow and Rayleigh-Bènard convection) should have maximums no larger than the noise. Unfortunately, this is not practical for reasons that will be explained next, and fortunately it is often not necessary. For example, the length scale of the computational noise could be much smaller than the relevant length scale of interest for studying the geometry of the dynamics. In this case, a comparison of the maximum consecutive distance in the sample to the diameter of the point cloud in Per is often useful. For instance, if a point cloud has diameter 100 and the smallest relevant length scale for the geometry to be studied is 10 , then a maximum consecutive distance of 10 is sufficient for the sampling of the time series, even if the noise threshold is on length scale 1 . Thus, it is the interplay of these three numbers that determine if one has sampled a continuous time series densely enough.

Evaluating these three quantities from an initial time sample may indicate that an increase in the sample rate is required to resolve the dynamics at the relevant spatial scale. In the context of a large-scale computation such as that required for the 3D simulation of Rayleigh-Bénard convection, it is easier to save the data at a higher sampling frequency than to develop numerical methods that save data based on an adaptive time step. In Section 10. we demonstrate the approach introduced here using approximately $7 \times 10^{5}$ equally-spaced snapshots of the temperature field of Rayleigh-Bénard convection. It should be immediately apparent that the set $X$ is too large to compute the associated persistence diagrams $\operatorname{PD}\left(X, d_{\star}\right)$, for $\star \in B, W^{2}, W^{1}$, directly. The first step would require computing the distance matrix for $X$, which would involve $49 \times 10^{10}$ distance computations. Note, however, that using a fast sampling rate leads to collecting unnecessarily many samples at the places where the dynamics are slow. This suggests that an appropriate choice of down-sampling will allow us to capture the global geometry of the point cloud.

Definition 7.2. Let $X$ be a point cloud in a metric space $(M, d)$. Fix $\delta>0$. A set $Y \subseteq X$ is a $\delta$-dense subsample of $X$ if for every $x \in X$, there exits a $y \in Y$ such that $d(x, y)<\delta$.

The following theorem [28] guarantees that using a $\delta$ dense subsample enables us to detect geometric features with life span larger than $\delta$.

Theorem 7.3. Let $X$ be a point cloud in a metric space $(M, d)$ and $Y$ a $\delta$-dense subsample of $X$. Then $d_{B}(\operatorname{PD}(X, d), \operatorname{PD}(Y, d))<\delta$.

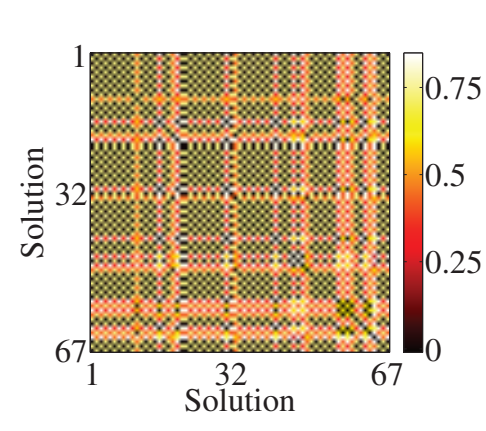

(a)

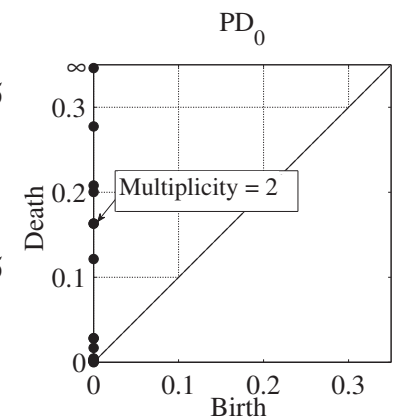

(b)

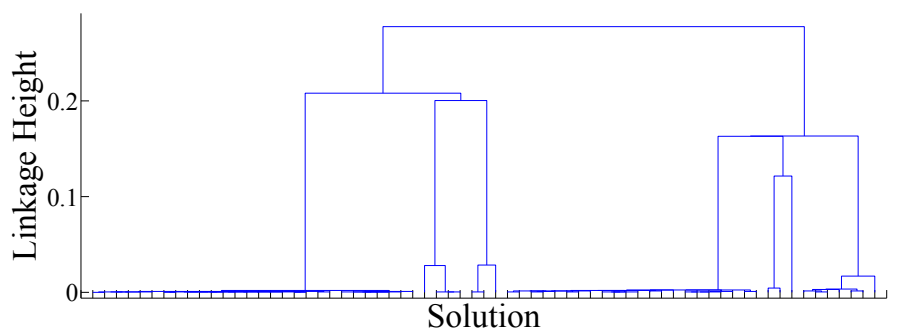

(c)

Figure 15: (a) Pairwise $d_{B}$ distances between the EQ and REQ points in $X=\left\{\operatorname{PD}\left(\omega_{n}\right) \mid n=1, \ldots, 67\right\}$ of the Komogorov flow found at $R e=26.43$ using Newton's method. (b) Corresponding persistence diagram $\mathrm{PD}_{0}\left(X, d_{B}\right)$ shows a clear gap between the points with death value $\theta_{d}=0.1215$ and $\theta_{d}=0.0285$. We interpret this gap as the separation between the signal and noise. (c) Dendrogram for the EQ and REQ points with the pairwise distances given by the matrix shown in part (a), with values halved to show the correspondence to the persistence diagram $\mathrm{PD}_{0}\left(X, d_{B}\right)$ in (b). The dendrogram indicates the presence of seven clusters with separation the same size as the above-mentioned gap.

Remark 7.4. According to the above theorem, there exists a bijection between the points in $\operatorname{PD}(Y, d)$ and $\operatorname{PD}(X, d)$ such that the distance between matched points is less than $\delta$. Furthermore, it can be shown that there is a bijection $\gamma: \operatorname{PD}(Y, d) \rightarrow \operatorname{PD}(X, d)$ with the following property: if $\gamma\left(\theta_{b}, \theta_{d}\right)=\left(\theta_{b}^{\prime}, \theta_{d}^{\prime}\right)$ and $\theta_{b}^{\prime} \neq \theta_{d}^{\prime}$, then $0 \leq \theta_{b}^{\prime}-\theta_{b}<\delta$ and $0 \leq \theta_{d}^{\prime}-\theta_{d}<\delta$.

To optimize the computational cost, we wish to choose a subsample of the point cloud $Y$ as small as possible. A point cloud $Y$ is $\delta$-sparse if, for every pair of distinct points $y_{1}, y_{2} \in Y$, the distance $d\left(y_{1}, y_{2}\right) \geq \delta$. Given a point cloud $X$ and a value $\delta \geq 0$, a $\delta$-dense and $\delta$-sparse subsample $Y$ may always be constructed [29]. Due to the size of the point cloud $X$ and the complexity of computing $d_{\star}$ for $\star \in B, W^{2}, W^{1}$, we use an alternate algorithm [28], which takes advantages of parallel computing structures and metric trees to construct a subsampling which is both $\delta$-dense and $\delta$-sparse .

\section{Distinguishing Equilibria}

We now apply the ideas presented in Section 7.1 to the problem of clustering symmetry-related equilibria of the 
Kolmogorov flow at $R e=26.43$. As discussed in Section 2.1, we sample a turbulent trajectory, shown in Figure 1(b). To obtain the EQ and REQ solutions, we use a Newton method. The initial guesses for the Newton method are the vorticity fields $\omega$ that are local minima of the $L^{2}$ norm of $\partial \omega / \partial t$. In this way, we find a collection $X=\left\{\operatorname{PD}\left(\omega_{n}\right) \mid n=1, \ldots, 67\right\}$ of persistence diagrams of vorticity fields corresponding to EQ and REQ of the Kolmogorov flow. These 67 solutions may be related to one another through any composition of the coordinate transformations listed in Section 2.1. Hence, it is non-trivial to determine how many unique classes of solutions there are and which solutions belong to which class. To perform this analysis, we use persistent homology.

We start by analyzing $\mathrm{PD}_{0}\left(X, d_{B}\right)$. The pairwise distances between the points in $X$ are shown in Figure 15)(a). As is discussed in Section 5, the distance between persistence diagrams of vorticity fields related by symmetry is small, while persistence diagrams corresponding to the vorticity fields that are not symmetry related differ by a larger amount. This implies that we can reformulate the question of identifying symmetry classes of equilibria as a clustering problem.

The persistence diagram $\mathrm{PD}_{0}\left(X, d_{B}\right)$, depicted in Figure 15(b), shows a clear gap between the persistence point with death value $\theta_{d}=0.0285$ and the persistence point with death value $\theta_{d}=0.1215$. We interpret this gap as separation between the signal and noise (numerical errors). Indeed, 0.0285 is just twice the estimate of the lower bound on numerical errors for the Kolmogorov flow obtained in Section 6. There are 7 points in $\mathrm{PD}_{0}\left(X, d_{B}\right)$ with death coordinate greater than 0.12 , and so we conclude that there are seven distinct symmetry classes of solutions.

The same conclusions can be achieved using a dendrogram based on single-linkage clustering. Figure 15 (c) shows the dendrogram for the different EQ a REQ points with the pairwise distances given by the distance matrix shown in Figure 15 (a), with values halved to show the correspondence with $\mathrm{PD}_{0}\left(X, d_{B}\right)$. Clearly, there are again seven different clusters with distances larger that 0.12 .

We validate the results of the persistence homology analysis by performing clustering using the Fourier amplitudes as follows. If $\hat{\omega}\left(k_{x}, k_{y}\right)$ is the Fourier amplitude of a mode $\left(k_{x}, k_{y}\right)$, then a translation of the pattern in the $x$ or $y$ directions in real space merely adds to the phase of $\hat{\omega}\left(k_{x}, k_{y}\right)$, leaving the magnitude unchanged. Hence, by comparing the amplitudes of the Fourier modes we could group vorticity fields which are related by translations. Since the conjugate modes $\hat{\omega}\left( \pm k_{x}, \pm k_{y}\right)$ relate fields which are related by inversion, to group the vorticity fields which are related by a combination of inversion and translation, we sum the amplitudes of the conjugate modes. Adding the amplitudes of conjugate modes yields a "reduced matrix," which is unique for all the vorticity fields related by the coordinate transformations that leave Equation (2) invariant. This approach also yields 7 distinct classes.

An analysis of $\mathrm{PD}_{0}\left(X, d_{W^{p}}\right), p=1,2$, yields the same results. There are several gaps between the death values of the points in the persistence diagrams. Again one of the gaps starts at roughly twice the value of the estimated lower bound of the noise. However, the separation is less pronounced. As discussed in Section [6, the $d_{W^{p}}$ metrics capture all the differences between the persistence diagrams, and the local numerical errors are summed together. Thus, a large number of small errors can obscure the distinction between the signal and noise.

\section{Stable Periodic Orbit of the Kolmogorov Flow}

In the previous section, we demonstrated the practicality of using persistent homology to cluster equilibria that are symmetry-related. In this section, we extend these ideas to the setting of recurrent orbits in the context of the Kolmogorov flow.

As is indicated in Figure 1(a), the projection of the orbit onto the real parts of the three dominant eigenvectors suggests a periodic orbit that is undergoing a slow drift in the direction of the continuous symmetry. The nature of this drift is reinforced by tracking this orbit in the space of persistence diagrams; since persistent homology is invariant under the continuous symmetry, this type of drift is not present in Per. As a result, we expect the time series to lie on a closed loop in Per. This is consistent with the information provided by the distance matrix of Figure 9] in which the dark lines parallel to the diagonal indicate that the distance between persistence diagrams becomes very small at regular time intervals.

For the remainder of this section, we use the ideas of Section 7.2 to verify that a circle provides a good description of geometry of the point cloud $X \subset$ Per generated by the time series sampled from the Kolmogorov flow. More precisely, we show that there is a single dominant feature in $\mathrm{PD}_{0}\left(X, d_{B}\right)$ and a single dominant feature in $\mathrm{PD}_{1}\left(X, d_{B}\right)$, which agrees with the persistent diagrams for a circle.

There are two issues that need to be considered: the first is the size of the data set, and the second is the spacing between the data points. As is indicated in Section 7, we use the Vietoris-Rips complex to compute persistent homology of point clouds. We remark that given $N$ data points, the full Vietoris-Rips complex has $2^{N}$ cells. Considering this, we complete our analysis with the distance matrices corresponding to $d_{B}, d_{W^{1}}$, and $d_{W^{2}}$ for 500 points, or roughly three periods of the Kolmogorov flow. In the next section, we introduce techniques for computing persistence on larger point clouds, which could arise due to increased sampling rates, sampling more periods, or both.

Since we are sampling from a single continuous trajectory, the fact that $\mathrm{PD}_{0}\left(X, d_{B}\right)$, as shown in Figure 16(a), suggests the existence of a single component does not come as a surprise. The persistence diagrams for $\operatorname{PD}_{0}\left(X, d_{W^{p}}\right)$, $p=1,2$, yield similar results and are not shown. However, it is worth noting that this is not a foregone conclusion as the location of and spacing between the points of the 


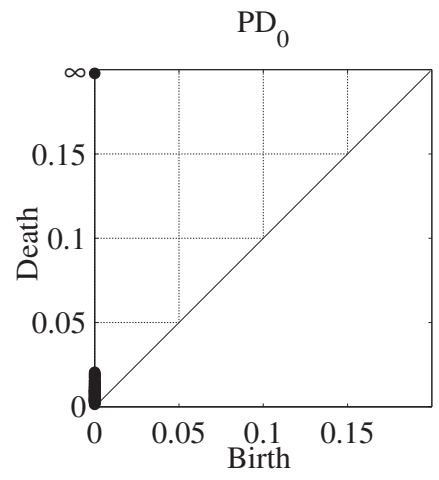

(a)

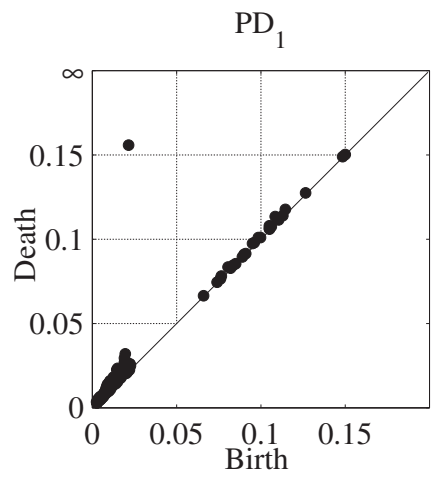

(b)
Figure 16: (a) The persistence diagram $\mathrm{PD}_{0}\left(X, d_{B}\right)$ for Kolmogorov flow at $R e=26.43$. Since all points with finite death coordinates die before 0.025 , there is only a single dominant point. (b) The persistence diagram $\mathrm{PD}_{1}\left(X, d_{B}\right)$, showing the single dominant generator at $(0.0215,0.1559)$

time series are dependent upon the speed along the periodic orbit. As is clear from Figure 9(a), the speed of the trajectory is not constant. However, it is fairly smooth, thus we do not expect extreme differences in the spacings between points.

As discussed in Section 7.3 we compare the noise threshold, $\theta=0.01$ (Table 1), to the maximum consecutive sample distance, $d_{B}=0.0654$ (Figure 9(a) caption), and the diameter of the point cloud, 2.64 (Figure 10(a)). The maximum consecutive sample distance is more than six times larger than the length scale of the noise for this system. However, the diameter of the point cloud is more than forty times larger than the consecutive sample distance. Thus, features on the length scale of one fortieth of the diameter of the entire point cloud will be resolved, which is sufficiently small to consider this an adequate sampling. We will return to this issue in the next section.

As indicated in Figure 16(b), the persistence diagram $\mathrm{PD}_{1}\left(X, d_{B}\right)$ clearly detects a single dominant loop along which the data is organized. Thus, we conclude that in Per, equipped with the metric $d_{B}$, the point cloud $X$ generated by the time series forms a loop with a minimal radius of 0.1344 . Table 3 shows the coordinates of the persistence point with the longest life span, its life span, and the second longest life span for each of the persistence diagrams $P D_{1}\left(X, d_{\star}\right), \star \in B, W^{2}, W^{1}$. As the table indicates, the life span of the dominant point is an order of magnitude larger than the next longest life span in each case, and so there is a single dominant feature in $\mathrm{PD}_{1}\left(X, d_{\star}\right)$. Additionally, note that the second longest life spans are as small or smaller than the lower bounds on numerical errors indicated by the first row of Table 1

\begin{tabular}{c|lcc}
\hline $\mathrm{PD}_{1}$ & $\begin{array}{l}\text { Dominant } \\
\text { coordinate }\end{array}$ & Max life span & $2^{\text {nd }}$ largest life span \\
\hline$d_{B}$ & $(0.022,0.156)$ & 0.134 & 0.013 \\
$d_{W^{2}}$ & $(0.075,0.405)$ & 1.366 & 0.105 \\
$d_{W^{1}}$ & $(0.703,2.069)$ & 0.330 & 0.016
\end{tabular}

Table 3: The coordinate of the dominant point in the persistence diagram $\mathrm{PD}_{1}\left(X, d_{\star}\right)$ for $\star=B, W^{2}, W^{1}$, its life span, and the second largest life span.

\section{Almost-Periodic Orbit of Rayleigh-Bénard Convection}

As mentioned in Section 7.3 , characterizing the geometry of a continuous trajectory becomes a challenge in the setting of dynamics with multiple time scales. To demonstrate this, we consider the numerical simulation of Rayleigh-Bénard convection, where from multiple perspectives it appears that the trajectory is close to a periodic orbit and that the rate of change in the patterns of the temperature field is far from constant. This can be clearly seen visually (see video 6,7 , or 8 in the supplementary materials). Moreover, both the speed plot, Figure 9(b), and the distance matrix, Figure 10(b), suggest recurrent dynamics. However, we note that the rate of change, especially using the bottleneck distance, is typically small except for short periods of time at which the speed spikes. The distance matrix has a distinct checkerboard pattern, with the edges corresponding to the spikes, again indicating a rapid and large change in location in the space of persistence diagrams.

The maximum bottleneck distance between the consecutive sampling points is 83.5 (Figure 9(b) caption), while the diameter of the point cloud is only $d_{B}=99.5$ (Figure 10(b)). Therefore, we expect that significant portions of the trajectory are missing. Indeed, Figure 17(a) shows that there are several persistence points in $\mathrm{PD}_{0}\left(X, d_{b}\right)$ with a (finite) death coordinate larger than ten. Thus, at a length scale of 20 (which is forty times larger than the noise threshold), the sample of the trajectory is broken into several pieces. The largest gap between different pieces of the trajectory is 40 , as indicated by the persistence point with coordinates $(0,20)$. This means that the sampling rate is far from adequate.

The diagram $\mathrm{PD}_{1}\left(X, d_{B}\right)$ in Figure 17(b) contains a single dominant point at $(20,32.5)$ with life span 12.5 . However, unlike in our analysis of the Kolmogorov flow in the previous section, we cannot argue that this point corresponds to a single dominant loop along which the data is organized because of the gaps in the sampling of the orbit. As mentioned in Section 7.3 the missing parts of the orbit could introduce loops of similar size corresponding to secondary structures. These structures might occur due to the fact that the loop corresponding to the underlying almost-periodic dynamics might be twisted, pinched, or bent in Per. In order to obtain information about secondary structures, we require a faster sampling rate. 


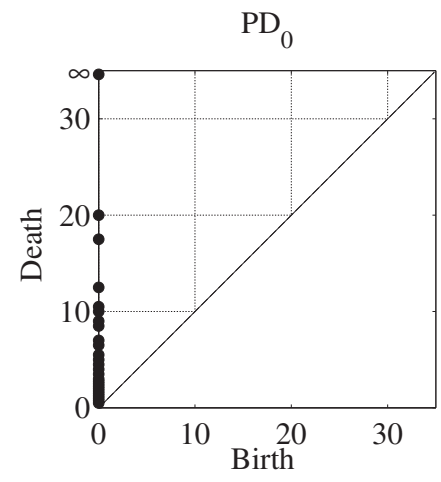

(a)

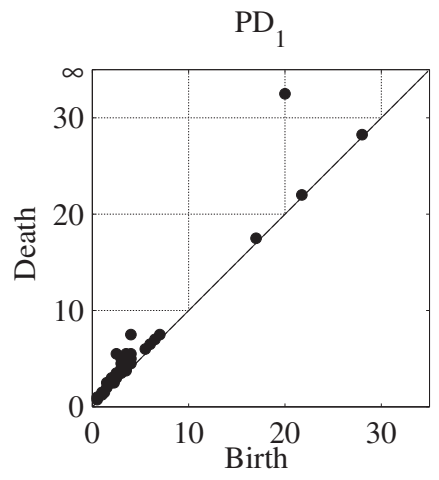

(b)

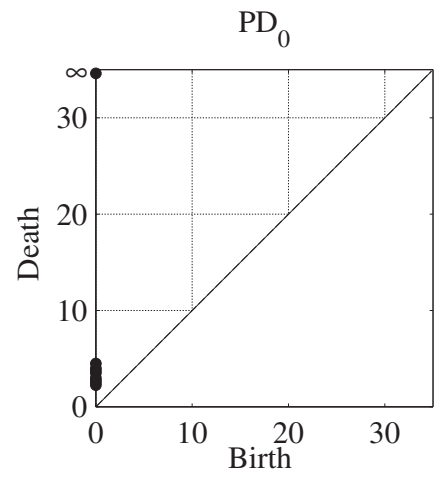

(a)

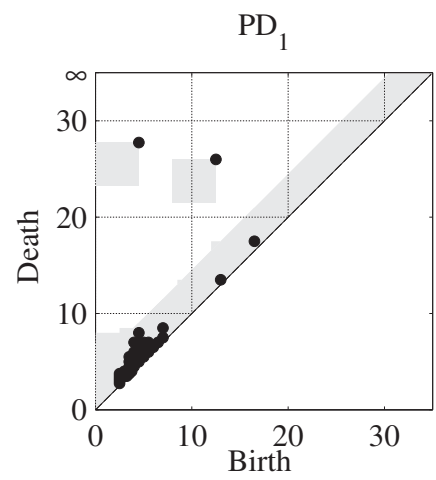

(b)

Figure 17: Persistence diagrams for 500 points of RayleighBénard convection, generated from the distance matrix shown in Figure 10 b). (a) The diagram $\mathrm{PD}_{0}\left(X, d_{B}\right)$ shows the appearance of persistence points with death values significantly greater than the noise threshold, indicating that the sampled trajectory is broken into pieces and sampling is not fast enough to resolve the periodic orbit. (b) The diagram $\mathrm{PD}_{1}\left(X, d_{B}\right)$ shows the presence of a loop that is born when the pieces of the orbit merge together.

We increased the sampling rate considerably and collected approximately $7 \times 10^{5}$ equally-spaced snapshots of the temperature field over four-and-a-half periods and compute the associated persistence diagrams, producing a point cloud $Y \subset$ Per. The maximal distances between the consecutive frames for the increased sampling rate drop to $d_{B}=4, d_{W^{1}}=28$, and $d_{W^{2}}=6.52$. The new value of $d_{B}$ is much closer to our estimate of the numerical error $(e=0.5)$ and it is more than 24 times smaller than the diameter of the point cloud generated from the slower sampling. Since the point cloud could only increase in diameter through increasing the sample rate, we consider this sampling rate to be satisfactory.

Our next step is to use the ideas introduced in Section 7.3 to reduce the size of the sample and to complete our analysis. First we construct a $\delta$-dense, $\delta$-sparse subsample $Y^{\prime}$ of the point cloud $Y$. The smallest value of $\delta$ for which we were able to compute the persistence diagrams $\mathrm{PD}\left(Y^{\prime}, d_{B}\right)$, using $256 \mathrm{~GB}$ of memory, is $\delta=4.5$. This value is only slightly larger than the largest distance between the consecutive states and, since the diameter of the subsampled point cloud is 99.5, the relationship between the length scale of the smallest detectable feature and the length scale of the diameter of the point cloud is still sufficient to resolve the geometry of the dynamics. The resulting persistence diagrams $\operatorname{PD}\left(Y^{\prime}, d_{B}\right)$ are shown in Figure 18

As shown by $\mathrm{PD}_{0}\left(Y^{\prime}, d_{B}\right)$, Figure $18(\mathrm{a})$, the point cloud merges to a single connected component at $\theta=4.5$. This indicates that the sample of the trajectory is not broken into different pieces separated from each other. Since the maximum consecutive distance between any two points in $Y$ is 4, the loop along which the data is organized should be present for $\theta=2$. However, after subsampling, it is possible that the loop will not be born until $\theta=2+\delta$.

Figure 18: Persistence diagrams for 70,000 points of Rayleigh-Bénard convection subsampled with $\delta=4.5$, resulting in a point cloud $Y^{\prime} \subset$ Per with 523 points. (a) Persistence diagram $\mathrm{PD}_{0}\left(Y^{\prime}, d_{B}\right)$ indicating a single dominant component above the noise threshold. (b) Persistence diagram $\operatorname{PD}_{1}\left(Y^{\prime}, d_{B}\right)$ with subsampling error bounds shaded in gray.

Looking at the diagram $\mathrm{PD}_{1}\left(Y^{\prime}, d_{B}\right)$ in Figure 18(b), we see that it contains a dominant point at $(4.5,27.75)$, and so the loop was indeed born before $\theta=2+\delta$. This is the loop along which the point cloud is organized. Now, there is another point, $(12.5,26) \in \mathrm{PD}_{1}\left(Y^{\prime}, d_{B}\right)$, with life span 13.5. This point corresponds to a secondary structure of the orbit. Indeed, it can be seen from the distance matrix for the $\delta$-sparse, $\delta$-dense subsample (not shown for brevity) that the part of the orbit corresponding to the fast dynamics (missing for the slow sampling rate) revisits very similar states before continuing along the main loop. However, the development of more sensitive tools is required to fully understand these secondary features.

We now turn our attention to the differences between the persistence diagrams of the original point cloud $Y$ and its subsample $Y^{\prime}$. Theorem 7.3 implies that $d_{B}\left(\operatorname{PD}\left(Y, d_{B}\right), \operatorname{PD}\left(Y^{\prime}, d_{B}\right)\right) \leq \delta$, and so there exists a bijection between the points in $\operatorname{PD}\left(Y, d_{B}\right)$ and $\operatorname{PD}\left(Y^{\prime}, d_{B}\right)$ such that the distance between matched points is less than 4.5. According to Remark 7.4 for the dominant point $(4.5,27.75) \in \mathrm{PD}_{1}\left(Y^{\prime}, d_{B}\right)$, there is exactly one corresponding point in $\operatorname{PD}_{1}\left(Y, d_{B}\right)$. This point is the unique point in $\mathrm{PD}_{1}\left(Y, d_{B}\right)$ that lies inside of the shaded box touching the point $(4.5,27.75)$, see Figure 18(b). The same is true for the other dominant point. Moreover, there are no points in $\mathrm{PD}_{1}\left(Y, d_{B}\right)$ outside of the shaded regions. Points in $\mathrm{PD}_{1}\left(Y, d_{B}\right)$ that do not correspond to the off-diagonal points in $\mathrm{PD}_{1}\left(Y^{\prime}, d_{B}\right)$ can appear only $\delta / \sqrt{2} \approx 3.18$ far away from the diagonal.

\section{Conclusion}

We have shown how persistent homology can be used to identify equilibria and study periodic dynamics, and how this method is particularly natural when solutions must be identified that lie on a group orbit. We study two regimes 
in Kolmogorov flow: chaotic dynamics due to the appearance of unstable fixed points, and a periodic flow that exhibits drift in a direction of continuous symmetry. We also study an almost-periodic orbit from Rayleigh-Bénard convection. We solve for the unstable equilibria in the first case and sample the periodic orbits in the other two cases, and use persistent homology to project these solutions to the space of persistence diagrams. We provide theoretical results that show this projection is stable with respect to numerical errors and discuss how the projection naturally identifies symmetry-related solutions. We give three different metrics on the space of persistence diagrams that can be used to study pattern evolution on large versus small spatial scales, and how these metrics can be used to estimate numerical error in the space of persistence diagrams. We develop an intuition for studying dynamics in the space of persistence diagrams by looking at point clouds in two-dimensional Euclidean space, and discuss methods for determining if a continuous trajectory has been sampled densely enough to resolve the underlying dynamics, as well as mathematical methods used to address issues associated with computing on large sample sets. We demonstrate the efficacy of these methods on Kolmogorov flow and Rayleigh-Bénard convection, comparing our methods to traditional Fourier methods where appropriate. Our results show that the geometry of the dynamics are recovered in each case. For Rayleigh-Bénard convection in particular, we show that the dynamics are recovered even after truncating the simulated data to an 8-bit temperature field, and so this approach is suitable for studying data collected experimentally, rather than numerically. Also for this flow, we recover more subtle aspects of the geometry in the space of persistence diagrams. In summary, we have shown that this method is both robust to noise and sensitive to more complicated dynamics, and that it is appropriate for studying dynamics on datasets obtained experimentally. Our ongoing research will further refine these tools.

\section{Acknowledgments}

The work of MK, RL, and KM has been partially supported by NSF grants NSF-DMS-0835621, 0915019, 1125174, 1248071, and contracts from AFOSR and DARPA. The work of JRF, BS and MFS has been partially supported by NSF grants DMS-1125302, CMMI-1234436. The work of MRP and MX has been supported by NSF grant DMS-1125234.

\section{Appendix A. Homology of Sets on a Torus}

Topologically, a torus $T=S^{1} \times S^{1}$ is a closed surface defined as the product of two circles. It can be also described as a quotient of the Cartesian plane under the identifications $(x, y) \sim(x+1, y) \sim(x, y+1)$. The homology groups of $T$ are given by

$$
H_{n}(T)= \begin{cases}\mathbb{Z} & \text { if } n=0,2, \\ \mathbb{Z}^{2} & \text { if } n=1, \text { and } \\ 0 & \text { otherwise. }\end{cases}
$$

Intuitively, this means that $T$ has a single connected component $(n=0)$, two independent loops $(n=1)$, and a single cavity $(n=2)$. For a more detailed treatment of the following material, see See Hatcher, Ch 0 for a reference to homotopies of maps, and Hatcher Ch 1 for a reference on identifying independent loops in a space, or the notion of the fundamental group.

In this section, we explain the notion of independent loops of subsets of a torus. By a loop, we mean a continuous path $\gamma:[0,1] \rightarrow T$ such that $\gamma(0)=\gamma(1)$. We will also be using the notion of a homotopy of loops, which can be thought of as deforming one loop continuously to another. More precisely, a homotopy of loops from a loop $\gamma_{0}$ to a loop $\gamma_{1}$ is a continuous function $F:[0,1] \times[0,1] \rightarrow T$ such that $F(s, 0)=\gamma_{0}(s)$ and $F(s, 1)=\gamma_{1}(s)$ for all $s \in[0,1]$, and $F(0, t)=F(1, t)$ for all $t \in[0,1]$. Define $-\gamma(t):=\gamma(1-t)$, which runs the loop $\gamma$ backwards in time, and define $n \gamma(t):=\gamma(\lfloor n t\rfloor)$, which traverses the loop $\gamma n$ times. Finally, given two loops $\alpha_{1}$ and $\alpha_{2}$, we can form their sum $\alpha_{1}+\alpha_{2}$ by taking a path $\delta:[0,1] \rightarrow T$ such that $\delta(0)=\alpha_{1}(0)$ and $\delta(1)=\alpha_{2}(0)$ and form the loop

$$
\left(\alpha_{1}+\alpha_{2}\right)(t)=\left\{\begin{array}{lr}
\alpha_{1}(4 t) & : t \in[0,1 / 4] \\
\delta(4 t-1) & : t \in[1 / 4,1 / 2] \\
\alpha_{2}(4 t-2) & : t \in[1 / 2,3 / 4] \\
-\delta(4 t-3) & : t \in[3 / 4,1]
\end{array}\right.
$$

Algebraically, this can be written as $\alpha_{1}+\delta+\alpha_{2}-\delta=\alpha_{1}+$ $\alpha_{2}$. We say that a loop $\gamma$ is independent of a collection of loops $\alpha_{1}, \ldots, \alpha_{k}$ if there does not exist a homotopy of loops from $\gamma$ to a linear combination of the loops $\alpha_{1}, \ldots, \alpha_{k}$.

Figure 19 shows eight subsets $\left\{X_{i}\right\}_{i=0}^{7}$ of a torus. Note that $X_{i} \subset X_{j}$ for $i<j$, and the sets can be considered as sub-level sets of some scalar function $f$. We will now examine each set and identify the independent loops in each.

The set $X_{0}$ is contractable. Hence, every loop inside $X_{0}$ can be deformed to a point inside of $X_{0}$. This means that there is no independent (nontrivial) loop present in this set.

The set $X_{1}$ cannot be contracted to a point. It forms a band that wraps around the torus. There are many different loops (wrapping once around the torus from left to right in the picture) inside of this band. However, we can choose a single loop $\alpha_{1}$ that represents all of them; every other loop can be either continuously deformed to a linear combination of the loop $\alpha_{1}$, or contracted to a point. Similarly, the set $X_{2}$ contains two independent loops.

The set $X_{3}$ is formed by linking the horizontal bands present in $X_{2}$. The loops $\alpha_{1}$ and $\alpha_{2}$ are still independent in $X_{3}$ (one cannot be deformed to the other inside $X_{3}$ ). It 
might seem that there is a new independent loop, $\gamma$. However, this is not case because $\gamma$ can be deformed (inside of $X_{3}$ ) to the union of the black lines corresponding to $\alpha_{1}, \alpha_{2}$ and $\delta$. After this deformation, the loop traverses $\delta$ twice: the right part of the deformed loop traverses $\delta$ from the top to the bottom, and the left part in the opposite direction. Algebraically, the deformed loop can be expressed as $\alpha_{1}-\delta-\alpha_{2}+\delta=\alpha_{1}-\alpha_{2}$. This shows that $\gamma$ can be deformed to a linear combination of the loops $\alpha_{1}$ and $\alpha_{2}$. Thus, $\gamma$ is not a new independent loop.

The set $X_{4}$, obtained from $X_{3}$ by adding another link between the horizontal bands, contains a new independent loop, $\beta_{1}$, consisting of the edges $\delta_{1}, \delta_{2}, \delta_{3}$ and $\delta_{4}$ ( $\left.\beta_{1}=\delta_{1}+\delta_{2}+\delta_{3}+\delta_{4}\right)$. This means that the loop $\beta_{1}$ cannot be deformed inside of $X_{4}$ to a linear combination of the loops $\alpha_{1}$ and $\alpha_{2}$. Again, the loop $\gamma$ is not independent from $\alpha_{1}, \alpha_{2}$, and $\beta_{1}$ because it can be perturbed to $\alpha_{1}+\delta_{3}-\alpha_{2}+\delta_{4}-\alpha_{2}+\delta_{1}+\delta_{2}=\alpha_{1}-\alpha_{2}+\beta_{1}$, which is a linear combination of the loops $\alpha_{1}, \alpha_{2}$, and $\beta_{1}$. Therefore, there are three independent loops in this case. Adding another link between the horizontal bands creates another independent loop. Hence, the number of independent loops for two bands with $n$ links is $n+1$.

Alternatively, we can view the set $X_{3}$ as a single band with one puncture, and $X_{4}$ as a single band with two punctures. The number of independent loops is $n+1$, where $n$ is the number of punctures, and the extra loop is generated by the band.

Due to the identification $(x, y) \sim(x, y+1)$, the set $X_{5}$ contains another link between the horizontal bands. This band creates another puncture. In Figure 19(f), this puncture seems to have four distinct components (white blocks in the corners). However, under the boundary identification, they correspond to a single component. Therefore, there are four independent loops.

The independent loops start disappearing as the punctures are filled in. The set $X_{6}$ contains a single puncture, and according to the previous argument, there are two independent loops, $\alpha$ and $\beta$. In this case, the loop $\gamma$ can be deformed to a point inside of the set $X_{6}$. Finally, the set $X_{7}=T=S^{1} \times S^{1}$ contains two independent loops corresponding to the two copies of $S^{1}$ that generate the torus.

\section{Appendix B. Computational aspects}

The computations involved in this paper were performed on a cluster computer with 896 cores. The computation of the persistence diagrams for the states of the systems as well as computation of the distance matrices have very low memory requirements (not more than a few GB). The only memory intensive computations involve generating persistence diagrams for rips complex representing the point cloud in the space of persistence diagrams. For this purpose, we used a machine with $260 \mathrm{~GB}$ of available memory. This computation was performed using a single CPU. The reason for not providing the exact comput- ing times in this paper is two fold: 1) Computing times tend to vary between the images of the same size depending on the complexity of the pattern, and therefore they could be misleading. Similarly, the time it takes to compute the distance matrices is very sensitive to the distribution of the points in the underlying persistence diagrams. 2) Perseus software, used for our computations, is implemented in a way that makes the mathematics involved very clear. Unfortunately, this is not the most efficient computationally, and therefore its performance lacks considerably when compared to the latest implementations 30] such as DIPHA 31] and PATH [32]. Our code for computing distance matrices is also not completely optimized. Moreover, there are new arbitrage algorithms for computing distances which highly outperform the classical algorithms. In this paper, we intended to introduce the new techniques rather than provide the reader with exact computation times, which are likely to be completely out of date very soon.

\section{References}

[1] M. Gameiro, K. Mischaikow, and W. Kalies, "Topological characterization of spatial-temporal chaos," Phys. Rev. E, vol. 70, Sept. 2004.

[2] M. Gameiro, K. Mischaikow, and T. Wanner, "Evolution of pattern complexity in the Cahn-Hilliard theory of phase separation," Acta Materialia, vol. 53, pp. 693-704, Febr. 2005.

[3] M. Kramar, A. Goullet, L. Kondic, and K. Mischaikow, "Quantifying force networks in particulate systems," Physica D, vol. 283, no. 0, pp. $37-55,2014$.

[4] K. Krishan, H. Kurtuldu, M. F. Schatz, M. Gameiro, K. Mischaikow, and S. Madruga, "Homology and symmetry breaking in Rayleigh-Bénard convection: Experiments and simulations," Phys. Fluids, vol. 19, Nov. 2007.

[5] H. Kurtuldu, K. Mischaikow, and M. F. Schatz, "Measuring the departures from the Boussinesq approximation in RayleighBénard convection experiments," J. Fluid Mech., vol. 682, pp. 543-557, 2011.

[6] H. Kurtuldu, K. Mischaikow, and M. F. Schatz, "Extensive scaling from computational homology and Karhunen-Loève decomposition analysis of Rayleigh-Bénard convection experiments," Phys. Rev. Lett., vol. 107, no. 3, 2011.

[7] H. Edelsbrunner and J. L. Harer, Computational topology. Providence, RI: American Mathematical Society, 2010. An introduction.

[8] G. Carlsson, "Topology and data," Bull. Am. Math. Soc. (N.S.), vol. 46, no. 2, pp. 255-308, 2009.

[9] S. Weinberger, "What is... persistent homology?," Not. Am. Math. Soc., vol. 58, no. 01, pp. 36-39, 2011.

[10] P. Cvitanovi, D. Borrero-Echeverry, K. M. Carroll, B. Robbins, and E. Siminos, "Cartography of high-dimensional flows: A visual guide to sections and slices," Chaos, vol. 22, no. 4, pp. -, 2012 .

[11] G. Kawahara, M. Uhlmann, and L. van Veen, "The significance of simple invariant solutions in turbulent flows," Annual Review of Fluid Mechanics, vol. 44, no. 1, pp. 203-225, 2012.

[12] E. C. G. Adcock, Aaron; Carlsson, "The ring of algebraic functions on persistence bar codes," eprint arXiv:1304.0530, p. 21, 2013.

[13] V. I. Arnold and L. D. Meshalkin, "Seminar led by A. N. Kolmogorov on selected problems of analysis (1958-1959)," Usp. Mat. Nauk, vol. 15, no. 247, pp. 20-24, 1960.

[14] B. Suri, J. Tithof, R. Mitchell, R. O. Grigoriev, and M. F. Schatz, "Velocity profile in a two-layer Kolmogorov-like flow," Phys. Fluids, vol. 26, 2014. 


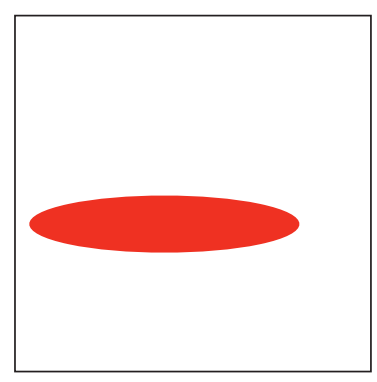

(a) $X_{0}$

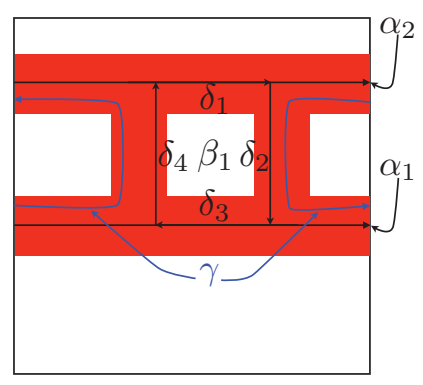

(e) $X_{4}$

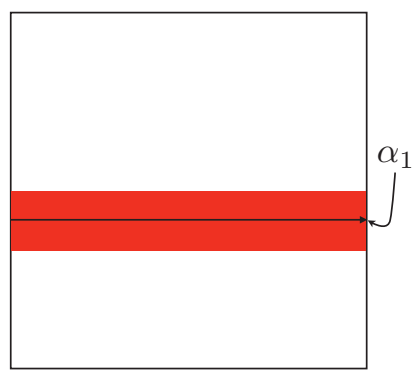

(b) $X_{1}$

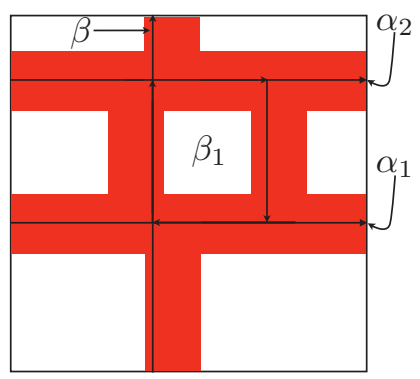

(f) $X_{5}$

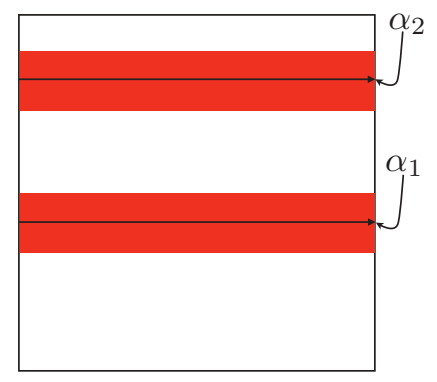

(c) $X_{2}$

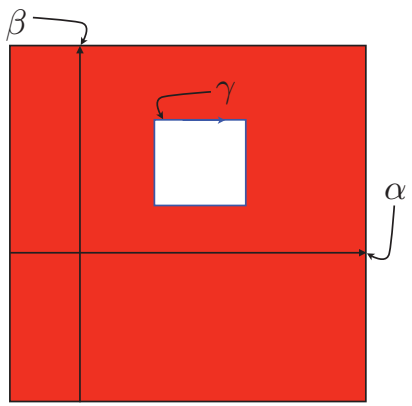

(g) $X_{6}$

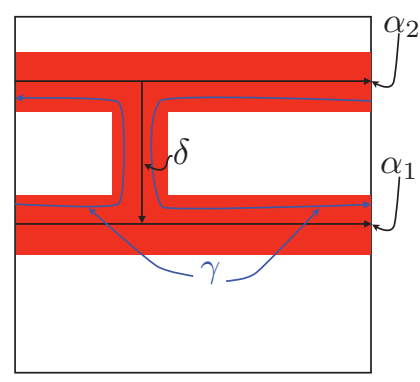

(d) $X_{3}$

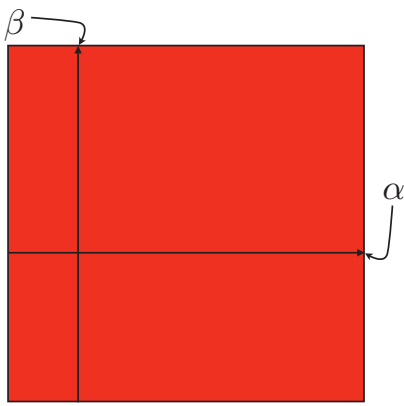

(h) $X_{7}$

Figure 19: Different subsets of the torus. (a) The set $X_{0}$ does not contain any independent loops because any loop in $X_{0}$ can be deformed to a point inside of $X_{0}$. (b) The set $X_{1}$ contains a single independent loop $\alpha_{1}$. All the other loops can be either deformed to $\alpha_{1}$ or to a point inside of $X_{1}$. (c) The set $X_{2}$ has two independent loops $\alpha_{1}$ and $\alpha_{2}$. (d) The loop $\gamma$ is not independent in $X_{3}$ because it can be deformed to the linear combination of the loops $\alpha_{1}$ and $\alpha_{2}$. (e) Adding an extra link creates a new independent loop $\beta_{1}$ in $X_{4}$. Again $\gamma$ is not independent because it can be deformed to a linear combination of the other loops. (f) The set $X_{5}$ is produced by adding another link which produces one new loop. (g) The loop $\gamma$ in $X_{6}$ is not independent because it can be deformed to a point. (f) $X_{7}=T$ contains two independent loops corresponding to two copies of $S^{1}$ generating the torus. 
[15] R. L. Panton, Incompressible flow. John Wiley \& Sons, 2006.

[16] R. Mitchell, Transition to turbulence and mixing in a quasitwo-dimensional Lorentz force-driven Kolmogorov flow. PhD thesis, Georgia Institute of Technology, 2013.

[17] G. J. Chandler and R. R. Kerswell, "Invariant recurrent solutions embedded in a turbulent two-dimensional Kolmogorov flow," J. Fluid Mech., vol. 722, pp. 554-595, 42013.

[18] M. C. Cross and P. C. Hohenberg, "Pattern formation outside of equilibrium," Rev. Mod. Phys., vol. 65, no. 3, p. 851, 1993.

[19] E. Bodenschatz, W. Pesch, and G. Ahlers, "Recent developments in Rayleigh-Bénard convection," Annu. Rev. Fluid Mech., vol. 32, no. 1, pp. 709-778, 2000.

[20] M. Paul, K.-H. Chiam, M. Cross, P. Fischer, and H. Greenside, "Pattern formation and dynamics in Rayleigh-Bénard convection: numerical simulations of experimentally realistic geometries," Physica D, vol. 184, no. 1, pp. 114-126, 2003.

[21] T. Kaczynski, K. Mischaikow, and M. Mrozek, Computational homology, vol. 157 of Applied Mathematical Sciences. New York: Springer-Verlag, 2004.

[22] K. Mischaikow and V. Nanda, "Morse theory for filtrations and efficient computation of persistent homology," Discret. \& Comput. Geom., vol. 50, pp. 330-353, Sept. 2013.

[23] "Perseus." http://www.math.rutgers.edu/ vidit/perseus.html April 2015.

[24] F. Chazal, L. J. Guibas, S. Y. Oudot, and P. Skraba, "Scalar field analysis over point cloud data," Discret. \& Comput. Geom., vol. 46, no. 4, pp. 743-775, 2011.

[25] F. Chazal, V. De Silva, and S. Oudot, "Persistence stability for geometric complexes," Geometriae Dedicata, vol. 173, no. 1, pp. 193-214, 2014.

[26] F. Chazal, V. De Silva, M. Glisse, and S. Oudot, "The structure and stability of persistence modules," arXiv preprint arXiv:1207.3674, 2012.

[27] P. Bubenik and J. A. Scott, "Categorification of persistent homology," Discrete \& Comput. Geom., vol. 51, no. 3, pp. 600627, 2014.

[28] S. Harker, R. Levanger, M. Kramár, and K. Mischaikow, "Capturing geometry of a large point cloud with confidence," In preparation.

[29] T. K. Dey, J. Sun, and Y. Wang, "Approximating loops in a shortest homology basis from point data," in Proc. TwentySixth Annu. Symposium Comput. Geom., pp. 166-175, ACM, 2010.

[30] U. T. P. G. H. A. H. N Otter, M. A. Porter, "A roadmap for the computation of persistent homology," arXiv:1506.08903, 2015.

[31] U. Bauer, M. Kerber, and J. Reininghaus, Distributed Computation of Persistent Homology, ch. 3, pp. 31-38. 